\title{
A combined Globo H-OCT4-T7 immunotherapy restrains colorectal tumor growth
}

\author{
Jiangyao Xu ( $\nabla 754753008 @ q q . c o m)$ \\ shenzhen university \\ Maixian Liu \\ shenzhen university \\ Shikang Liu \\ shenzhen university \\ Kan Liu \\ Shenzhen university \\ Zijun Ouyang \\ shenzhen university \\ Guangyi Jin \\ Shenzhen University \\ Desheng Lu \\ Shenzhen University \\ Wenxiao Jiang \\ Shenzhen University \\ Xingsheng Shu \\ Shenzhen University \\ Gaixia Xu \\ Shenzhen University \\ Yang Li \\ Shenzhen University \\ Christina C. N. Wu \\ Shenzhen University \\ Meiyi Chen \\ Shenzhen University \\ Guimiao Lin \\ Shenzhen University \\ Xiaomei Wang \\ Shenzhen University
}

Research 


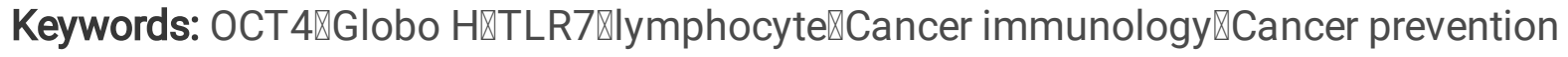

Posted Date: January 19th, 2021

DOI: https://doi.org/10.21203/rs.3.rs-147864/v1

License: (c) (1) This work is licensed under a Creative Commons Attribution 4.0 International License. Read Full License 


\section{Abstract}

Background: Malignant tumors express multiple common markers that might constitute targets for tumor immunotherapy.

Methods: Here we performed antigen epitope screening and molecular docking to design a new combination vaccine based on covalently linking Globo $\mathrm{H}$ and Oct 4 with a TLR7 agonist. We mainly use flow cytometry to analyze the changes in lymphocyte differentiation after the vaccine is applied. Use Elisa technology to detect the antibodies produced by the vaccine and the changes in lymphokines. In order to supplement the immunological data, RNA sequencing was performed on untreated and vaccinetreated tumors to define the interference of the vaccine on the genome-wide gene expression profile.

Results: Our results demonstrated that the Globo H-OCT4-T7 conjugate vaccine effectively induced cellular uptake, maturation and antigen presentation of BMDCs. The combination vaccine enhanced cytokine production, CTLs activity elicited high levels of IgG. Importantly, the tumor immune microenvironment was significantly improved in tumor-bearing mice treated by Globo H-OCT4-T7 conjugate vaccine, demonstrated by increased the ratio of M1 to M2 TAMs, reduced number of Tregs in PBMCs and promoted the infiltration of tumor-specific IFN $y$-producing CD8+ T cells. Prophylactic vaccination of Globo H-OCT4-T7 significantly reduced tumor growth by $>80 \%$ in both CT26 transplanted mice and in a human PDX mouse model when exposing mice to the combined vaccine compared to control. Our novel, combination vaccine is safe and effective at preventing tumor growth in mice.

Conclusions: This combination vaccine is more effective than the single vaccine, and thus has the potential to prevent OCT4 and Globo H from expressing cancer.

\section{Background}

Cancer cells can suppress the immune system's ability to effectively recognize and kill tumor cells by inducing immune tolerance $(1,2)$. Tumor immunotherapy is a treatment that controls and clears tumors by restarting and maintaining the tumor-immune cycle and restoring the body's normal anti-tumor immune response. Such immunotherapies include monoclonal antibody-type immunological checkpoint inhibitors such as PD-1/PD-L1 inhibitors and CTLA-4 inhibitors (3-5), therapeutic antibodies such as Panitumumab $(6-8)$, cancer vaccines such as Provenge $(9,10)$, cell therapy such as CAR-T $(11-14)$, and small molecule inhibitors. The past decade has seen numerous reports of positive results from these tumor immunotherapies, with strong anti-tumor activity elicited in the treatment of various cancers, such as melanoma, non-small cell lung cancer, kidney cancer and prostate cancer (15). Cancer stem cells (CSCs) are very few cell with self-renewal ability and multiple differentiation potential in tumor tissue (16). A large number of researches show that after radiotherapy and chemotherapy, tumor resistant cells can regenerate and express CSCs markers, which is highly invasive and closely related to tumor recurrence and metastasis. Therefore CSCs will be one of the important drug targets in the treatment of malignant tumors (17). At the same time, due to the expression of normal stem cell protein, the immune 
system cannot effectively recognize it, resulting in strong immune escape ability of CSCs and high clinical recurrence rate of tumor patients. At present, the preventive vaccines against CSCs are generally aimed at a single target, such as ALDH (aldehyde dehydrogenase) (18), CD44 (19), etc., while, multi-target CSCs vaccines are rarely reported. CSCs express different markers in different periods. For a single target CSCs preventive vaccine, it can only target CSCs in a specific period, and cannot effectively eliminate the variant CSCs. There is still a need for new therapy scheme for CSC possessing targeted, sustained and systemic therapeutic effects, to be able to fight against metastasis and recurrence, while limiting offtarget effects. We hope to improve the killing power of CSCs in different periods by preparing multi-target vaccine. The concept of multi-target vaccination is to limit the escape ability of tumors by targeting more potential redundancies.

OCT4 (octamer-binding transcription factor 4) is a member of the mammalian POU transcription factor family; it is a key regulator of embryonic stem cell self-renewal and pluripotency $(20,21)$. OCT4 is highly expressed in breast, testicular and bladder cancers, germ cell tumors and cancer stem cells (22-25). Tumors with high OCT 4 expression are associated with advanced disease progression, high risk of metastasis, and short cancer-related survival time (26). OCT4-specific T cells are found at a high frequency $(80 \%)$ in T cells of PBMC isolated from healthy donors compared to newly diagnosed patients with germ cell tumors (35\%), suggesting that reducing the OCT4 immune response might lead to germ cell tumorigenesis and tumor development $(27,28)$. Because OCT4 is highly expressed in tumor cells, but it is either absent or very lowly expressed in many normal cells, it might constitute a potential tumor stem cell biomarker and an ideal target for tumor therapy. Indeed, previous studies have reported that vaccines targeting OCT4 can have significant therapeutic effects $(29,30)$.

Globo $\mathrm{H}$ is a hexasaccharide glycosphingolipid that was originally discovered in the human MCF-7 breast cancer cell line $(31,32)$. Globo $\mathrm{H}$ is overexpressed in most malignancies and its high levels might be predictive of poor patient outcomes (33-35). Globo $\mathrm{H}$ is generally not expressed in normal tissues, with only weak expression at the secretion boundary and other intraluminal sites that are not accessible to the immune system $(36,37)$. As such, Globo $\mathrm{H}$ has also become an attractive vaccine target for epithelial tumors. Synthetic Globo H, which binds to the keyhole limpet hemocyanin (KLH), has been used as a therapeutic vaccine in clinical trials to elicit an antibody response (38-41) and additional studies are ongoing to elucidate the effectiveness of Globo $\mathrm{H}$ immunotherapy in cancer patients.

Adjuvant is an effective substance to enhance immunogenicity and immune protection of vaccines (42, 43). TLRs are a family of proteins expressed in various cells, including immune cells (dendritic cells [DCs], macrophages, B cells and T cells). TLR signaling is activated by ligands and contributes to the host immune defenses $(44,45)$. Of the 13 TLRs, only TLR7/8 recognizes GU-rich short single-stranded RNA (ssRNA) and synthetic small molecules, such as nucleoside analogs and imidazoquinolin, which have been reported to have immunostimulatory properties and have been used in many tumor vaccines (46). This ability creates an opportunity to screen and modify highly potent TLR7 agonists to enhance the immune response. In addition, TLR7/8 agonists not only activate antigen-presenting cells (APCs) but also promote the activation of T cells and natural killer cells $(N K)(47,48)$. Activation of TLR-7 results in the 
involvement of MyD88, MAL, IRAKs, and IKKa, which promote activation of IRF and production of type 1 IFNs and pro-inflammatory cytokines (49-52). Because most tumor cells express high levels of tumorspecific antigens, TLR7 agonists have attracted widespread interest in tumor immunotherapy (53).

Because of tumor heterogeneity, vaccines containing a combination of antigens may improve efficacy and cover a wider range of cancer targets Here, we introduced a novel TLR7 agonist called SZU-101 (54) and demonstrated that OCT4 antigenic peptides are covalently linked to Globo H polysaccharides and TLR7 agonists (Globo H-OCT4-T7), which have a surprising anti-tumor effect on colorectal cancer(CRC) by inducing a tumor-specific immune response. The mechanism by which this combination vaccine promotes an immune response was investigated.

\section{Materials And Methods}

\section{Epitope prediction and molecular docking}

The OCT4 protein sequence was obtained from the National Center for Biotechnology Information (NCBl; http://www.ncbi.nlm.nih.gov). Using the epitope prediction program IEDB (http://www.iedb.org/), the OCT 4 sequence was analyzed and peptides with high affinity for MHC class I was identified. The peptide sequence was: EAAGSPFSGGPVSFPLAPGPHFGTPGYGSPHF. The efficacy of this peptide fragment at suppressing mouse teratocarcinoma cell growth has been reported by our groups recently (Cancer Biol Med 2020. doi: 10.20892/j.issn.2095-3941). Discovery studio software (55) was used to design the optimal combination of Globo H-OCT4-T7 vaccines by combining energy and scoring functions.

\section{Experimental animals, cell lines and human colorectal cancer specimens}

Female BALB/c mice (4-6 weeks old) and female BALB/c nude mice (4-6 weeks old) were purchased from the Medical Laboratory Animal Center (Guangzhou, Guangdong Province, China). All animal treatments and experimental protocols for this study were performed with the approval of the Laboratory Animal Welfare and Ethics Committee, School of Medicine, Shenzhen University (Permit No. AEWC-

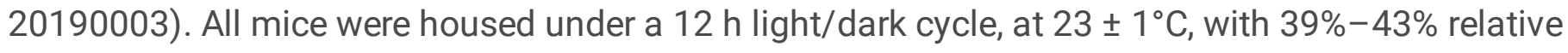
humidity. Water and food were provided ad libitum. CT26 cells (mouse colorectal cancer) and F9 (mouse teratocarcinoma cells) were maintained in Dulbecco's modified Eagle's medium (DMEM) with $10 \%$ fetal bovine serum (FBS) and $100 \mathrm{U} / \mathrm{mL}$ penicillin-streptomycin (all from Hyclone Laboratories, Inc., South Logan, UT, USA). Colorectal carcinoma samples $(n=16)$ were obtained from patients at Shenzhen People's Hospital in China. Data collected for each patient included their age, sex and tumor samples (Tables 1). All clinicopathological information was obtained by reviewing pathology reports. This study and the data obtained from clinical samples were approved by the Ethics Committee of Shenzhen People's Hospital.

\section{Vaccine preparation and administration}

The OCT4 peptides were synthetized at ChinaPeptides Co., Ltd. (Shanghai, China). The Globo H in the form of lyophilized powder was bought from Oligotech (Crolles, French). EDC (\#22980, Thermo Fisher 
Scientific) and NHS (\#24500, Thermo Fisher Scientific) were used to activate carboxylic acid as an active ester. The molar ratio of Globo H, OCT4 peptides, TLR7 agonist, EDC and NHS was 1:1:2.5:5:5 and this mixture was incubated at $4^{\circ} \mathrm{C}$ overnight. The final conjugate Globo H-OCT4-T7 was stable for at least 6 months at $-20^{\circ} \mathrm{C}$ at concentration of $1.5 \mu \mathrm{M}$.

\section{Generation and stimulation of bone marrow derived dendritic cells (BMDCs) in vitro}

BMDCs were isolated from hind limb bones of mice as previously described $(56,57)$. Briefly, both distal bone ends were excised and the marrow cells were flushed using RPMI 1640 (Gibco, Grand Island, NY). The red blood cells were lysed and the remaining cells were centrifuged at $450 \times \mathrm{g}$ for $10 \mathrm{~min}$. Then, 2 $' 10^{6} / \mathrm{mL}$ cells were aliquoted in RPMI 1640 supplemented with $10 \% \mathrm{FBS}, 2 \mathrm{mmol} / \mathrm{L} \mathrm{L-glutamine} 1$ $\mathrm{mmol} / \mathrm{L}$ sodium pyruvate, $100 \mathrm{U} / \mathrm{mL}$ penicillin, $100 \mathrm{mg} / \mathrm{mL}$ streptomycin, $20 \mathrm{ng} / \mathrm{mL}$ GM-CSF (PeproTech) and $10 \mathrm{ng} / \mathrm{mL} \mathrm{IL}-4$ (PeproTech) and incubated at $37^{\circ} \mathrm{C}$. The cells were cultured in six well plates in complete medium with cytokines at $4^{\prime} 10^{6}$ cells $/ 2 \mathrm{~mL} /$ well. On the fifth day of culturing, OCT4-T7, Globo $\mathrm{H}-\mathrm{T} 7$ or Globo H-OCT4-T7 were added to a final concentration of $10 \mathrm{mg} / \mathrm{mL}$, and the cells were cultured for two more days. On day 7, most of the non-adherent cells had acquired typical dendritic morphology, and these cells were used as the source of DCs in subsequent experiments. For flow cytometry, the cells were cultured for 7 days and then rinsed three times with PBS and briefly trypsinized to form a single cell suspension. The cells were stained for $30 \mathrm{~min}$ on ice with the following fluorescence-labeled antibodies: Anti-mouse MHCII APC (eBioscience: 17-5321-82), Anti-mouse CD86 PE (clone: GL1), Anti-mouse CD80 FITC (clone: 16-10A1). Finally, the cells were washed twice with cold PBS and analyzed on flow cytometry (BD FACSAria II cell sorter, BD Biosciences).

\section{Tumor protection in BALB/c mice}

Female BALB/c mice (6-10 mice per group) were vaccinated with untreated group (PBS), T7 (0.012

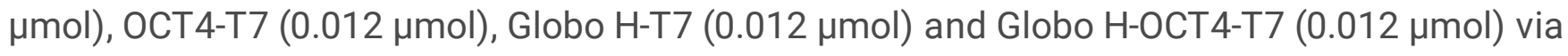
intraperitoneal (i.p.) injection on days 0,14 and 21. To assess the memory response, a booster dose was given on day 28. Five days after the final vaccination, the mice were challenged with $1^{\prime} 10^{5}$ CT26 cells (100 $\mu \mathrm{l}$ of cell mixture in 50\% Matrigel; \#354234, Corning) that were injected subcutaneously (s.c.) into the lower flank of the right back. Then, the tumor size was measured periodically with calipers. Starting from day 0 after cell inoculation, the tumor volume was measured every 2 days. The tumor size was calculated using the formula: $0.5 \times$ length $\times$ width ${ }^{2}\left(\mathrm{~cm}^{3}\right)(58)$. Mice were euthanized when the tumor size was $>1.5 \mathrm{~cm}^{3}$. Mouse blood samples were collected from retro-orbital tissues.

\section{Enzyme-linked immunosorbent assay (ELISA)}

Sera samples were collected at 3-5 days after four doses of the vaccine had been administered. An ELISA for multiple cytokines in peripheral blood was performed using a Ready-SET-GO! ELISA kit (eBioscience, Thermo Fisher Scientific), according to the manufacturer's instructions. 
Splenocytes and lymph nodes $(\mathrm{LN})$ of vaccinated mice were harvested for evaluating the cellular immune responses 4-5 days after the final vaccination. The cytotoxicity activity of the vaccinated mice was analyzed using a CytoTox96 Non-Radioactive Cytotoxicity Assay Kit (G1780, Promega, Madison, WI, USA). Briefly, splenocytes from the vaccinated mice were mixed at an effector: target (E: $T$ ) ratio of 12.5:1, 25:1, 50:1 with $5^{\prime} 10^{3}$ CT26 cells. Cytotoxic T lymphocyte (CTL) activity was evaluated by analyzing the released LDH. The cytolysis rate (\%) was calculated based on the equation: cytotoxicity $(\%)=$

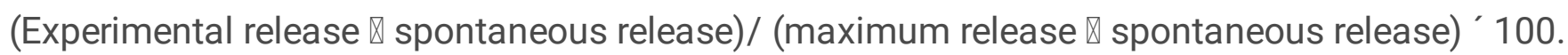

\section{Humoral immune response}

In order to assess humoral responses to anti-OCT4 IgG and anti-Globo H IgG levels, ELISA was performed to measure antigen-specific total IgG, according to previously described (59). Briefly, each 96-well ELISA plate (Costar, Corning, New York, USA) was coated with $2 \mu \mathrm{g}$ synthetic OCT4 peptide and $2 \mu \mathrm{g}$ Globo H and incubated at $4^{\circ} \mathrm{C}$ overnight. The plates were then washed with PBST ( $0.01 \mathrm{M}$ PBS containing $0.05 \%$ Tween 20, pH 7.4) three times. Serum samples were detected at a different dilution for $2 \mathrm{~h}$. Alkaline phosphate-conjugated detection antibody for total IgG (Sigma-Aldrich) was added and incubated for $1 \mathrm{~h}$ at room temperature. Then, p-NPP substrate (Millipore) and stop solution (50 $\mu \mathrm{L}$ of $3 \mathrm{M} \mathrm{NaOH}$ ) were added to each well, and the optical density (OD, $405 \mathrm{~nm}$ ) was measured using a spectrophotometer (BioTek, Winooski, VT, USA).

\section{Tumor-reactive IgG assay}

To detect tumor-reactive serum IgG, CT26 and F9 cells were cultured with serum harvested from BALB/c mice immunized with different vaccines for $4 \mathrm{~h}$, washed thoroughly with PBS, and stained with an GFPlabeled anti-mouse IgG antibody, before analysis by flow cytometry using a FACS Calibur flow cytometer (BD FACSAria II cell sorter, BD Biosciences).

\section{Splenocyte and lymph node cell proliferation assay}

One week after the last immunization, spleen and lymph node cells were isolated from BALB/c mice and plated into 96-well plates. The lymphocytes were then co-cultured with different vaccines. After 3 days, a 3-(4, 5-dimethylthiazol-2-yl)-2, 5-diphenyltetrazolium bromide (MTT) assay was performed to test the proliferation of the lymphocytes, as previously described (60).

\section{Analysis of tumor infiltrating immune cells}

CT26-bearing mice were sacrificed on day 28. Tumors were dissociated using a mouse tumor dissociation kit according to the manufacturer's protocol (Miltenyi Biotec). The cells were stained for PE F4/80 (macrophage marker), APC CD86 (M1 marker), PE/Cy7 CD206 (M2 marker). Post-staining, the cells were washed and quantified by flow cytometry. The ratio of M1/M2 macrophages was calculated based on the $\%$ M1 positive cells to $\%$ M2 positive cells. Tregs were identified as $C D 4^{+}$CD2 $5^{+}$Foxp $3^{+}$.

\section{RNA sequencing}


Gene expression analysis of subcutaneous tumor samples from mice exposed to different vaccine immunizations was evaluated at Novo Gene Corporation (Beijing, China). The up-regulated or downregulated genes were identified by filtering the RNA-seq data with the following cutoff values: 2-fold change in expression level and a false discovery rate (FDR) analog with a $P$ value of $<0.05$.

\section{H\&E and immunofluorescence staining}

Tumor tissues were harvested and fixed in $10 \%$ buffered formalin, dehydrated, cleared and embedded in paraffin according to standard procedures (61). The tissues were then sectioned at $3 \mu \mathrm{m}$, mounted onto polylysine-coated slides and then the sections were stained with hematoxylin and eosin (H\&E). Histopathological morphology was checked under a light microscope (Olympus BX51). For immunofluorescence assays, the sections were deparaffinized in xylene and rehydrated in graded ethanol. Antigen retrieval was performed in citrate buffer $(\mathrm{pH} 6.0)$ by boiling for $30 \mathrm{~min}$ in a microwave and cooling down to room temperature. Then, the sections were blocked with $3 \%$ bovine serum albumin in PBS for $1 \mathrm{~h}$ at room temperature. Following this, the sections were stained with antibodies against mouse CD8 (14-0081-82, Invitrogen), OCT4 (701756, Invitrogen) and SSEA3 (Globo H homologs) (1:200, 14-8833-80, eBioscience) at $4^{\circ} \mathrm{C}$ overnight. After washing, the sections were incubated with a secondary AlexaFluor 488 goat anti-rabbit antibody (1:200, ab16669, Abcam) for $1 \mathrm{~h}$, and were then washed and mounted in Fluoroshield with DAPI (F6057, Sigma-Aldrich). Images were captured under a Nikon Eclipse E400 microscope using an Olympus DP73 camera and analyzed with cellSence Entry software.

\section{The transfer experiment}

The primary tumor was resected from a patient diagnosed with CSC. For the patient-derived xenograft (PDX) model, the fresh patient tumor specimen was cut into small pieces (less than 2-3 $\mathrm{mm}$ ) and initially implanted subcutaneously in nude BALB/c mice. For the transfer experiment, PBMCs harvested from healthy people's peripheral blood were stimulated with or without Globo H-OCT4-T7 vaccines and injected (i.p.) into the PDX once a week for 3 weeks. The tumor volumes were measured every one week.

\section{TUNEL staining}

Tissue sections were dewaxed with xylene and then hydrated with a gradient of ethanol using the TUNEL FITC Apoptosis detection kit (yazyme, A111). After washing three times with PBS, it was permeabilized with proteinase $\mathrm{K}$. The TUNEL reaction mixture was prepared, and after completion of the reaction, the film was sealed with a DAPI-containing sealing liquid, and then observed under a fluorescence microscope.

\section{Statistical analysis}

We used Graphpad Prism 8.0 to conduct statistical analyses. For lifespan, Kaplan-Meier survival was utilized and $P$ values were calculated using the log-rank test. To compare two groups, Student's t-test was performed. One-way analysis of variance (ANOVA) with Duncan's test was conducted for comparing multiple groups. $P<0.05$ was considered statistically significant. 


\section{Results}

\section{A Globo H-OCT4-T7 combination vaccine is stable and has low binding energy and is suitable for use as an immunotherapy vaccine}

We first aimed to conjugate TLR7 agonist to peptides antigens or polysaccharide. Here, we combined the three components of Globo H-OCT4-T7 in different arrangements to produce multiple vaccine formats in different spatial conformations: type I, II III and IV. We pioneered the use of computer simulation technology to virtually screen the optimal spatial configuration based on the principle of lower bound energy. We chose C-type lectin (DC-SIGN), a receptor protein that DC cells recognize antigens, and then we used discovery studio software to perform 3D homology modeling DC-SIGN on DC cells. We molecularly docked four designed vaccine conformations with DC-SIGN, and calculated the binding energy to obtain a scoring function (Fig. 1A). The software can use a variety of scoring functions to give a score for the combination. A higher score indicates that the combination is better and more consistent with the real situation. From the scoring function, the comprehensive score of the type I vaccine was the highest (18.64) compared to the other three configurations (Fig. 1B). We know that when two things are combined, the lower the energy, the more stable it is. We then tested the binding energy of the four vaccines. From the combination score, the type II vaccine had the lowest binding energy and the most stable structure, but these parameters did not significantly differ from the other three configurations (Fig. 1C). After that the type 2 vaccine's spatial structure easily breaks the linked chemical bonds and makes synthesis difficult, we chose the type I vaccine as the subject of subsequent studies.

To construct the conjugate vaccines, we chose EDC and NHS to active carboxylic acid as active ester. We first used them to activate the carboxyl group of the TLR7 agonist, form an amide bond attached to OCT4, and then use OCT4 as a carrier to link Globo $\mathrm{H}$ through an amide bond at the other end to synthesize Globo H-OCT4-T7 (Fig. 1D). We also generated OCT4-T7 (MW 4613 Da), Globo H-T7 (MW $1757 \mathrm{Da}$ ), Globo H-OCT4-T7 (MW $5926 \mathrm{Da}$ ) and analyzed all three molecules by mass spectrometry (Figure 1E).

\section{Globo H-OCT4-T7 promotes antigen uptake in BMDCs and induces BMDC maturation}

The induction of protective immune responses involves several key steps. These include antigen uptake and processing by antigen-presenting cells (APCs), activation of APCs for effective T and B cells. After immunization, APCs, especially DCs, capture and present antigens through the $\mathrm{MHC}$ I and $\mathrm{MHC}$ II pathways to activate $T$ and $B$ lymphocytes. Having generated our multi-marker vaccine, we next examined its efficacy on antigen uptake in BMDCs. We incubated OCT4-T7, Globo H-T7 and Globo HOCT4-T7 with BMDCs for $24 \mathrm{~h}$ and quantified antigen uptake through surface binding by MFI of GFP and APC using flow cytometry. Cellular localization of OCT4 and Globo H was examined by confocal laser scanning microscopy (Fig. 2A). We found a 3-fold increase of Globo H and 4-fold increase of OCT4 uptake in BMDCs co-cultured with Globo H-OCT4-T7 compared to controls (Fig. 2B\&C). These results suggest that compared to OCT4-T7 and Globo H-T7, Globo H-OCT4-T7 can significantly increase antigen uptake of BMDCs, partly via enhanced surface binding. 
To investigate the effect of Globo H-OCT4-T7 on DCs in vitro, we harvested immature BMDCs on day 5 after isolation and exposed them to Globo H-OCT4-T7 for $48 \mathrm{~h}$. We then characterized DC maturation based on surface marker expression by flow cytometry. Here we found that exposure to Globo H-OCT4-T7 significantly enhanced $(P<0.01)$ the expression levels of CD80 and CD86 - surface markers that indicate DC maturation - compared with other groups (Fig. 2D). MHC II expression levels $(P<0.05)$ were also upregulated compared with T7 group in DCs treated with Globo H-OCT4-T7 (Fig. 2E). Taken together, these data indicate a facilitative role of Globo H-OCT4-T7 on DC maturation.

\section{Globo H-OCT4-T7 induces cytokine production and enhances cytotoxic T lymphocyte (CTL) responses against tumors}

To evaluate the efficacy of Globo H-OCT4-T7 as potential cancer vaccine, mice were immunized four times. One week after the forth immunization, we collected serum and then measured IFN-y, IL-12, IL-2 and TNF- $a$ cytokine levels by ELISA. We found that the levels of secreted IFN- $y$, IL-12, IL-2 and TNF- $a$ were significantly higher in the Globo H-OCT4-T7 vaccine group compared with the other groups (Fig. 3A). To test the efficacy of T cells in mediating tumor-specific CTL responses, we established an in vitro CTL assay. First, we co-cultured effector cells (splenocytes and lymph node cells isolated from immunized mice) with the CT26 expressing OCT4 and Globo H as target cells. We then tested target-cell lysis at the Effector: Target (E: T) cell ratios of 12.5:1, 25:1 and 50:1 (Fig. 3B). Importantly, the effector T cells isolated from mice immunized with Globo H-OCT4-T7 lysed 50\% target CT26 cells, while the lysed target CT26 cells in control group was only 20\% (Fig. 3B). This finding suggests that mice immunized with Globo H-OCT4-T7 can induce an efficient CTL response against the CT26 target cells.

Following the successful preparation and evaluation the immunogenicity of vaccines, we explored whether humoral immunity was involved in the anti-CRC effect. We found that the titer of OCT4 and Globo H-specific antibodies in the serum was significantly increased (Fig. $3 \mathrm{C}$ ). We also found that the level of tumor-specific IgG antibodies was significantly increased in mice immunized with Globo H-OCT4T7 vaccine compared to F9 cells expressing only OCT4 as a control (Fig. 3D\&E). These results indicated that the anti-tumor effect induced by the Globo H-OCT4-T7 vaccine was dependent on both cellular and humoral immunity.

\section{Globo H-OCT4-T7 vaccination has therapeutic effects in a CT26 tumor model}

To examine the antitumor efficacy of Globo H-OCT4-T7, we thus next evaluated the therapeutic effects of Globo H-OCT4-T7 vaccines using a protection experiment. Briefly, we vaccinated BALB/c mice with the combination vaccine following an immunization schedule (Fig. 4A), with PBS as a negative control. Then, we measured the tumor volumes every 2 days after the tumors began to grow. Compare with the PBS group, all treatment groups showed reduced tumor growth rates (Fig. 4B). As expected, the Globo H-OCT4T7 vaccine group exhibited the best anti-tumor effects with $\sim 5$-fold smaller tumor size compared to control group, suggesting the advantages of combination therapy (Fig. 4C). Furthermore, in an overall survival analysis after the final day of treatment, median survival analysis was also significantly prolonged up to 20 days in Globo H-OCT4-T7 vaccine group compared to control (Fig. 4D). These findings 
indicate that muti-target therapeutic strategies combining TLR7 agonist, OCT4 and Globo H surface polysaccharide can increase the immunotherapy effects of CT26 and has a long-lasting overall response.

\section{The Globo H-OCT4-T7 vaccine changes the proportion of macrophages in the tumor microenvironment and reduces the proportion of regulatory T cells in the PBMC}

The occurrence and metastasis of tumors are closely related to the internal and external tumor microenvironment. Tumor cells can promote tumor growth and development through autocrine and paracrine changes and maintain conditions for survival and development. Tumor-associated

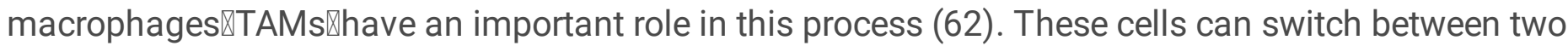
major phenotypes, the anti-tumor M1 phenotype and pro-tumor M2 phenotype, depending on surrounding signals [63]. We immunized BALB/c mice four times (as described above) and after 32 days, inoculated the mice subcutaneously with $1 \times 10^{5} \mathrm{CT} 26$ cells. After 1 week, we isolated TAM and found that the population of $\mathrm{CD}^{2} 6^{+} \mathrm{F} 4 / 80^{+} \mathrm{M} 1 \mathrm{TAMs}$ from BALB/c mice immunized with Globo H-OCT4-T7 vaccine increased compared to control $(P<0.05$, Fig. $5 A)$, while there was no difference in the population of $\mathrm{CD} 206^{+} \mathrm{F} 4 / 80^{+} \mathrm{CD} 45^{+} \mathrm{M} 2 \mathrm{TAM}$ cells among the other groups (Fig. 5B). The M1/M2 ratio was calculated by measuring the $\% \mathrm{M} 1$ positive cells to $\% \mathrm{M} 2$ positive cells. As shown in Fig. 5B, M1 macrophages were significantly upregulated to an M1 phenotype $(P<0.01)$ in comparison to control.

Meanwhile, Tregs play a key role in the maintenance of immune tolerance and participated in the inhibition of harmful immune responses to the host. Evidence suggested that Tregs were immunesuppressive lymphocytes, which helped immune escape and suppressed anti-tumor immune response $(63,64)$. So the number of $C D 3^{+} C D 4^{+} C D 25^{+}$Foxp3 Tregs cells in PBMCs from the tumor-bearing BALB/C mice were recorded and analyzed by Flow cytometry. We observed a significantly decreased $\mathrm{CD}^{+}{ }^{+} \mathrm{CD} 4^{+} \mathrm{CD} 25^{+}$Foxp3 Tregs population after Globo H-OCT4-T7 vaccine treatment when compared with control and T7 groups $(P<0.05$, Fig. 5C\&D). These data indicate that Globo H-OCT4-T7 vaccine acted on the TAMs and enhanced macrophage polarization and Tregs mediates the tumor inhibition.

\section{The tumor suppressive effect of Globo H-OCT4-T7 vaccine is relied on T-cell regulation}

We next aimed to identify the mechanism of action of Globo H-OCT4-T7 vaccine on tumor inhibition. Whole transcriptome RNA-seq analysis was carried out on tumors from PBS, T7, OCT4-T7, Globo H-T7 and Globo H-OCT4-T7 groups. The transcriptome heat map illustrated great differences between the treatment groups (Fig. 6A). From the transcriptome volcano map, the control group had 54 up-regulated genes and 351 down-regulated genes compared to the Globo H-OCT4-T7 group (Fig. 6B). We then analyzed the vaccine action pathway using ClueGO (Fig. 6C). Gene enrichment analysis showed that the three vaccines mainly affected the immune pathway within the tumor. Specifically, the differentially expressed genes between the Globo H-T7 and the control groups were mainly concentrated in the regulation of cell activation, side of membrane and regulation of leukocyte activation (Fig. 6D); the differentially expressed genes between the OCT4-T7 and the control group were mainly concentrated in the positive regulation of the immune response, side of membrane and T-cell activation (Fig. 6E); and the 
differentially expressed genes between the Globo H-OCT4-T7 group and the control group were mainly concentrated in the regulation of leukocyte activation, the adaptive immune response, and T-cell activation (Fig. 6F). Overall, transcriptome analysis showed that the tumor suppressive effect of the Globo H-OCT4-T7 vaccine is mainly focused on T-cell regulation.

\section{Globo H-OCT4-T7 induces antitumor immune responses}

It is well established that $\mathrm{CD} 8^{+} \mathrm{T}$ cells are important effector cells in vaccine-induced anti-tumor responses (65). Interestingly, our combination vaccine received a single component effect during the induction of $\mathrm{CD}^{+}, \mathrm{CD}^{+} \mathrm{T}$ cells differentiation. $\mathrm{CD} 8+\mathrm{T}$ cells in the spleen and $\mathrm{LN}$ of mice immunized with OCT4-T7 rather than Globo H-OCT4-T7 were significantly increased (Fig. 7A, B\&C). We consider this is due to $T$ cell proliferation. To this end, we examined cell proliferation by MTT assay and calculated the absolute number of $C D 8^{+} T$ cells in the spleen and LN, and found no significant difference between the Globo H-OCT4-T7 group and OCT4-T7 group (Fig. 7D).

Previous studies demonstrated that T-cell dysfunction or exhaustion in tumor-bearing mice hinders the production of anti-tumor immunity (66). To examine the potential role for preventing CD8 ${ }^{+} \mathrm{T}$-cell exhaustion with our combined vaccine, we examined the production of IFN- $\gamma$, which is the signature Th1 cytokine and granzyme, which can directly kill infected cells. We found that the combined vaccine elevated the production of IFN-y by $\mathrm{CD}^{+} \mathrm{T}$ cells (Fig. $7 \mathrm{~A}, \mathrm{~B} \& \mathrm{E}$ ). These results indicate that the combined vaccine can induce antitumor immune responses and prevent $\mathrm{CD} 8^{+} \mathrm{T}$ cells from $\mathrm{CT} 26$-induced exhaustion.

\section{Globo H-OCT4-T7vaccineincreases the number of $\mathrm{CD}^{+}$cells in the tumor microenvironment and promotes the apoptosis of tumor cells}

Tumor infiltrating lymphocytes (TIL) are a major component of the immune cells that infiltrate tumors. $\mathrm{TIL}$ contains different proportions of $\mathrm{CD} 3^{+} \mathrm{CD} 4^{+}$and $\mathrm{CD} 3^{+} \mathrm{CD} 8^{+} \mathrm{T}$ lymphocytes. Its functional status is considered to be an important indicator of positive prognosis and long-term survival of patients (67). Here, we aimed to further assess $C D 8^{+} T$ cell populations by histological analyses. We inoculated nude mice subcutaneously with $1 \times 10^{5} \mathrm{CT} 26$ cells after four rounds of immunization with the vaccine. Then, we isolated tumors when they became visible and monitored the proportion of $\mathrm{CD} 8^{+} \mathrm{T}$ cells by immunofluorescence. We found that the number of $\mathrm{CD} 8^{+} \mathrm{T}$ cells in the tumor tissue sections of mice immunized with Globo H-OCT4-T7 was significantly higher than those in other groups $(P<0.05$, Fig. 8 A\&B). Next, we examined tumor-cell apoptosis by TUNEL staining and found that the percentage of apoptotic tumor cells was significantly increased in tumor tissues immunized with Globo H-OCT4-T7 vaccine $(P<0.05$, Fig. $8 \mathrm{C} \& \mathrm{D})$. The TUNEL assay further demonstrated that Globo H-OCT4-T7 induced extensive tumor cell apoptosis.

Globo H-OCT4-T7 vaccine can safely inhibit tumor growth in PDX mice 
Safety evaluation is an important aspect in the development of immunoprevention. Blood cell levels of different vaccines demonstrated no signs of change compared with control healthy mice (Table S1). However, White blood cell count (WBC) and Lymphocytes (Lym) of T7 group showed a litter reduction compared with control $(P<0.05)$. The vital organs (heart, liver, spleen, lung and kidney) of the mice were taken out four times after immunization for toxicological analysis, and no significant difference was found between the groups, illustrating the safety of the vaccine (Fig. 9A).

We then assessed tumor samples from 16 patients by immunohistochemistry, to determine the positive rates of OCT4 and Globo H expression (Table.1). Here, we found that most patients express highly OCT4 and Globo H. Then, we isolated PBMCs from the healthy people's peripheral blood and stimulated the cells with or without vaccines before implanting immunodeficient mice with patient tissues to construct PDX model (Fig. 9D). On the 50th day after inoculation, all the mice were then sacrificed, and tumor tissues were resected and weighed (Fig. 9B\&C). The combination therapy significantly inhibited tumor growth and brought the inhibition rate up to $60 \%(P<0.01)$. Immunohistochemical fluorescence analysis showed the content of OCT4 positive cells and Globo $\mathrm{H}$ positive cells in the original patient tumor samples had markedly decreased compared to control (Fig. 9E\&F). These data clearly indicate that Globo H-OCT4-T7 vaccine possesses an excellent therapeutic efficacy in the PDX model.

\section{Discussion}

Non-invasive cancer prevention using anti-cancer vaccines has non-toxic potential to ensure protection due to the specificity of the immune response and its long-term memory (68). CRC as the third most commonly diagnosed and the second leading cause of cancer death worldwide (69), the treatment of CRC is still a huge clinical challenge, suggesting that identification of CRC-specific targets for prevention is urgently needed. Accumulating evidence has revealed the importance of CSCs in chemotherapy and recurrence (70). Because of tumor heterogeneity, vaccines containing a combination of antigens may improve efficacy and cover a wider range of cancer targets (71). In this study, we use a novel TLR7 agonist SZU-101 as an adjuvant for the Globo H-OCT4 combination vaccines. The results demonstrated that Globo H-OCT4-T7 induced specific cellular immune response and protection of BALB/c mice from CRCs growth, as evidenced by significantly enhanced lymphocyte proliferation, IFN-y, IL-2, IL-12, TNF-a, cytotoxicity and in vivo antitumor effects, compared with control groups and single antigen vaccine.

CSCs are key drivers of cancer recurrence after therapy, but there is currently a lack of immune vaccines that target these cells (72). The identification of specific markers for CSCs has been the focus of research, enabling the preparation of immune vaccines by stimulating an immune response to target and kill CSCs, thereby preventing the onset or recurrence of cancer $(73,74)$. Synthetic Globo $\mathrm{H}$ binds to the key pore limpet hemocyanin $(\mathrm{KLH})$ and has been used as a therapeutic vaccine in clinical trials to elicit an antibody response in breast cancer cells (75). Clinical trials are currently underway to test vaccines for specific markers $(76,77)$. However, the challenge remains that tumor cells differ from foreign microorganisms in that their specific proteins are less immunogenic, and the mechanism of tumor immune escape is complicated; notably, tumor cells are not easily recognized by the immune system. 
Targeting CSCs may damage tumor growth and decrease the risk of tumor recurrence and progression (78). We focused on CSCs as a target of cancer immunoprevention to achieve more efficient anti-tumor response. In this study, we illustrated that Globo H-OCT4-T7 significantly enhanced immune response. Elucidating the mechanism underlying these effects indicated that such combination therapy enhanced the $\mathrm{CD} 8+T$ cell population and enhancing $C T L$ activity.

We propose that coupling multiple active components together in one vaccine would have better therapeutic effect compared to a single component vaccine. Compared with conventional vaccines, peptide subunit vaccines have many advantages, such as minimal toxicity, easy preparation, immediate and long-term memory and cost effectiveness (79). Indeed, we demonstrate that our dual-target tumor vaccine based on polysaccharide peptide is more effective at killing tumor cells than the equivalent single-target vaccine. Therefore, we can also explore the overall effect of different ratios of different components of the vaccine on the efficacy of the combination vaccine.

\section{Conclusions}

In summary, our study first showed that Globo H-OCT4-T7 acted on TAMs, promoted their antigenpresenting functions, and thereby increased the infiltration of activated $\mathrm{CD} 8^{+} \mathrm{T}$ cells in tumors, spleen and lymph nodes. Overall, by combining our in vitro, in vivo and clinical analyses, we propose the efficacy of the combination vaccine, Globo H-OCT4-T7 as the basis of a potential viable CRC cancer vaccine in the future. This new vaccine was also outlicensed for clinical evaluation of $\mathrm{OCT} 4^{+} \mathrm{Globo} \mathrm{H}^{+}$expressing cancers, and for consideration of preventive application.

\section{Abbreviations}

\section{OCT4}

octamer-binding transcription factor 4

\section{Globo H}

hexasaccharide glycosphingolipid

PBMC

peripheral blood mononuclear cell

BMDC

Bone marrow-derived dendritic cells

\section{ALDH}

aldehyde dehydrogenase

TLR7

Toll-like receptor 7

CRC

Colorectal cancer

CSC

Cancer stem cells 
APC

antigenpresenting cell

NK

natural killer cell

TAM

Tumor-associated macrophages

PDX

Patient-Derived tumor Xenograft

$\mathrm{KLH}$

Keyhole limpet hemocyanin

ssRNA

Single-Stranded Ribonucleic Acid LN $\square$ Splenocytes and lymph nodes

CTL

Cytotoxic T lymphocyte

\section{Declarations}

\section{Contributions}

Jiangyao Xu, Maixian Liu, Shikang Liu, Tingting Chen and Kan Liu performed the experiments and analyzed data. Zijun Ouyang provided clinical specimen. Christina C. N. Wu, Dennis A. Carson conceived, wrote the manuscript and supervised the study. Xiaomei Wang designed or/and supervised this project and revised the manuscript. The author (s) read and approved the final manuscript.

\section{Ethics declarations}

Ethics approval and consent to participate

\section{Not applicable.}

Consent for publication

\section{Competing interests}

The authors declare no competing interests.

\section{Acknowledgements}

We would like to thank the Instrumental Analysis Center of Shenzhen University (Xili Campus) for their assistance in our experiments. We would also like to thank Prof. Xinchun Chen (Shenzhen University) for providing flow cytometry.

\section{Funding}


This work was supported from the National Natural Science Foundation of China (NSFC) (81803081, 81703050, 81772002), Shenzhen Basic Research Project (No. JCYJ20170818092553608, No. JCYJ20170307100703967, No. JCYJ20160331114230843, JCYJ20170817102634964), and China (Shenzhen Peacock Innovation Team Project, No. KQTD20140630100658078).

\section{References}

1. Zaini RG \& Al-Rehaili AA (2019) The Therapeutic Strategies of Regulatory T Cells in Malignancies and Stem Cell Transplantations. J Oncol 2019:5981054.

2. De Velasco MA \& Uemura H (2018) Prostate cancer immunotherapy: where are we and where are we going? Curr Opin Urol 28(1):15-24.

3. Gu L, et al. (2019) The safety and tolerability of combined immune checkpoint inhibitors (anti-PD1/PD-L1 plus anti-CTLA-4): a systematic review and meta-analysis. BMC Cancer 19(1):559.

4. Papouin B, Mussini C, De Martin E, \& Guettier C (2018) [Hepatic and digestive adverse events of immune checkpoint inhibitors (anti-CTLA-4 and, anti-PD-1/PD-L1): A clinico-pathological review]. Ann Patho/38(6):338-351.

5. Grimm MO, Oppel-Heuchel H, \& Foller S (2018) [Treatment with PD-1/PD-L1 and CTLA-4 immune checkpoint inhibitors : Immune-mediated side effects]. Urologe A 57(5):543-551.

6. Kim TW, et al. (2018) Impact of Emergent Circulating Tumor DNA RAS Mutation in PanitumumabTreated Chemoresistant Metastatic Colorectal Cancer. Clin Cancer Res 24(22):5602-5609.

7. Boeckx N, et al. (2018) Effect of Primary Tumor Location on Second- or Later-line Treatment Outcomes in Patients With RAS Wild-type Metastatic Colorectal Cancer and All Treatment Lines in Patients With RAS Mutations in Four Randomized Panitumumab Studies. Clin Colorectal Cancer 17(3):170-178 e173.

8. Freeman DJ, et al. (2012) Tumor penetration and epidermal growth factor receptor saturation by panitumumab correlate with antitumor activity in a preclinical model of human cancer. Mol Cancer 11:47.

9. Arlen PM \& Wood LV (2012) Prostate cancer vaccines: moving therapeutic vaccination forward in the post-Provenge era. Expert Rev Vaccines 11(3):287-302.

10. Brower V (2010) Approval of provenge seen as first step for cancer treatment vaccines. J Nat/ Cancer Inst 102(15):1108-1110.

11. Jain T, et al. (2019) Utilization of Chimeric Antigen Receptor (CAR) T Cell Therapy in Clinical Practice for Relapsed/Refractory Aggressive B cell non-Hodgkin Lymphoma: An Expert Panel Opinion from the American Society for Transplantation and Cellular Therapy. Biol Blood Marrow Transplant.

12. Bishop MR (2019) The case for CAR T-cell therapy in follicular lymphomas. Blood 134(7):577-578.

13. Jacobson C, Emmert A, \& Rosenthal MB (2019) CAR T-Cell Therapy: A Microcosm for the Challenges Ahead in Medicare. JAMA.

14. Frigault MJ, et al. (2019) Tisagenlecleucel CAR-T Cell Therapy in Secondary CNS Lymphoma. Blood. 
15. Botta GP, Granowicz E, \& Costantini C (2017) Advances on immunotherapy in genitourinary and renal cell carcinoma. Trans/ Cancer Res 6(1):17-29.

16. Batlle E \& Clevers H (2017) Cancer stem cells revisited. Nat Med 23(10):1124-1134.

17. Ge Y \& Fuchs $E$ (2018) Stretching the limits: from homeostasis to stem cell plasticity in wound healing and cancer. Nat Rev Genet 19(5):311-325.

18. Rodriguez-Torres M \& Allan AL (2016) Aldehyde dehydrogenase as a marker and functional mediator of metastasis in solid tumors. Clin Exp Metastasis 33(1):97-113.

19. Yan Y, Zuo X, \& Wei D (2015) Concise Review: Emerging Role of CD44 in Cancer Stem Cells: A Promising Biomarker and Therapeutic Target. Stem Cells Trans/ Med 4(9):1033-1043.

20. Chen Y, et al. (2019) OCT4B-190 protects against ischemic stroke by modulating GSK-3beta/HDAC6. Exp Neurol 316:52-62.

21. Soheili S, Asadi MH, \& Farsinejad A (2017) Distinctive expression pattern of OCT4 variants in different types of breast cancer. Cancer Biomark 18(1):69-76.

22. Ezeh UI, Turek PJ, Reijo RA, \& Clark AT (2005) Human embryonic stem cell genes OCT4, NANOG, STELLAR, and GDF3 are expressed in both seminoma and breast carcinoma. Cancer 104(10):22552265.

23. Wang $\mathrm{F}$, et al. (2019) Long non-coding RNA HOXA-AS2 promotes the migration, invasion and stemness of bladder cancer via regulating miR-125b/Smad2 axis. Exp Cell Res 375(1):1-10.

24. Iskender B, Izgi K, \& Canatan H (2016) Reprogramming bladder cancer cells for studying cancer initiation and progression. Tumour Bio/37(10):13237-13245.

25. Lee SR, et al. (2015) Activation of EZH2 and SUZ12 Regulated by E2F1 Predicts the Disease Progression and Aggressive Characteristics of Bladder Cancer. Clin Cancer Res 21(23):5391-5403.

26. Shen L, et al. (2014) High Expression of SOX2 and OCT4 Indicates Radiation Resistance and an Independent Negative Prognosis in Cervical Squamous Cell Carcinoma. J Histochem Cytochem 62(7):499-509.

27. Liu J, Wang L, Wang Y, Zhang W, \& Cao Y (2017) Phenotypic characterization and anticancer capacity of CD8+ cytokine-induced killer cells after antigen-induced expansion. PLoS One 12(4): $\mathrm{e} 0175704$.

28. Li W, et al. (2017) Dual inhibiting OCT4 and AKT potently suppresses the propagation of human cancer cells. Sci Rep 7:46246.

29. Park GB \& Kim D (2017) TLR5/7-mediated PI3K activation triggers epithelial-mesenchymal transition of ovarian cancer cells through WAVE3-dependent mesothelin or OCT4/SOX2 expression. Oncol Rep 38(5):3167-3176.

30. Lin G, et al. (2015) A conjugate of octamer-binding transcription factor 4 and toll-like receptor 7 agonist prevents the growth and metastasis of testis embryonic carcinoma. J Trans/ Med 13:166.

31. Chandrasekaran EV, Xue J, Neelamegham S, \& Matta KL (2006) The pattern of glycosyl- and sulfotransferase activities in cancer cell lines: a predictor of individual cancer-associated distinct 
carbohydrate structures for the structural identification of signature glycans. Carbohydr Res 341(8):983-994.

32. Dolo V, Adobati E, Canevari S, Picone MA, \& Vittorelli ML (1995) Membrane vesicles shed into the extracellular medium by human breast carcinoma cells carry tumor-associated surface antigens. Clin Exp Metastasis 13(4):277-286.

33. Dong WH, et al. (2016) Expression of key glycosphingolipid biosynthesis-globo series pathway genes in Escherichia coli F18-resistant and Escherichia coli F18-sensitive piglets. Anim Genet 47(4):428435.

34. Benktander J, Angstrom J, Breimer ME, \& Teneberg S (2012) Redefinition of the carbohydrate binding specificity of Helicobacter pylori BabA adhesin. J Biol Chem 287(38):31712-31724.

35. Accetta AC, et al. (2011) Type IV Borrmann gastric adenocarcinoma: analysis of curative resection results. Rev Col Bras Cir 38(4):237-244.

36. Chang WW, et al. (2008) Expression of Globo H and SSEA3 in breast cancer stem cells and the involvement of fucosyl transferases 1 and 2 in Globo H synthesis. Proc Natl Acad Sci U S A 105(33):11667-11672.

37. Ragupathi G, et al. (2005) Antibodies against tumor cell glycolipids and proteins, but not mucins, mediate complement-dependent cytotoxicity. J Immuno/ 174(9):5706-5712.

38. Zhou Z, Liao G, Mandal SS, Suryawanshi S, \& Guo Z (2015) A Fully Synthetic Self-Adjuvanting Globo H-Based Vaccine Elicited Strong T Cell-Mediated Antitumor Immunity. Chem Sci 6(12):7112-7121.

39. Hajmohammadi $M$, et al. (2015) Synthesis and characterization of a novel chemically designed (Globo)3-DTPA-KLH antigen. Drug Des Devel Ther 9:217-239.

40. Krug LM, et al. (2012) Immunization with N-propionyl polysialic acid-KLH conjugate in patients with small cell lung cancer is safe and induces IgM antibodies reactive with SCLC cells and bactericidal against group B meningococci. Cancer Immunol Immunother 61 (1):9-18.

41. Ragupathi $\mathrm{G}$, et al. (2008) Evaluation of widely consumed botanicals as immunological adjuvants. Vaccine 26(37):4860-4865.

42. Bastola R, et al. (2017) Vaccine adjuvants: smart components to boost the immune system. Arch Pharm Res 40(11):1238-1248.

43. Guimaraes LE, Baker B, Perricone C, \& Shoenfeld Y (2015) Vaccines, adjuvants and autoimmunity. Pharmacol Res 100:190-209.

44. Mifsud EJ, Tan AC, \& Jackson DC (2014) TLR Agonists as Modulators of the Innate Immune Response and Their Potential as Agents Against Infectious Disease. Front Immuno/ 5:79.

45. Chen WH, et al. (2012) Potential role for alternatively activated macrophages in the secondary bacterial infection during recovery from influenza. Immunol Lett 141(2):227-234.

46. Park CG, et al. (2018) Extended release of perioperative immunotherapy prevents tumor recurrence and eliminates metastases. Sci Trans/ Med 10(433). 
47. Kim H, et al. (2018) Acidic pH-responsive polymer nanoparticles as a TLR7/8 agonist delivery platform for cancer immunotherapy. Nanoscale 10(44):20851-20862.

48. Nouri-Shirazi M, Tamjidi S, Nourishirazi E, \& Guinet E (2018) TLR8 combined withTLR3 or TLR4 agonists enhances DC-NK driven effector Tc1 cells. Immunol Lett 193:58-66.

49. Martin PJ, et al. (2019) Cellular response and extracellular vesicles characterization of human macrophages exposed to fine atmospheric particulate matter. Environ Pollut 254(Pt A):112933.

50. Mandler WK, et al. (2019) Mouse pulmonary response to dust from sawing Corian(R), a solid-surface composite material. J Toxicol Environ Health A 82(11):645-663.

51. Ciaglia E, et al. (2019) Longevity-Associated Variant of BPIFB4 Mitigates Monocyte-Mediated Acquired Immune Response. J Gerontol A Biol Sci Med Sci.

52. Ceballos S, et al. (2018) Immunometabolic regulation by triterpenes of Eucalyptus tereticornis in adipose tissue cell line models. Phytomedicine 50:109-117.

53. Gigante M \& Ranieri E (2010) TLR7 and TLR8 as targets for tumor immunotherapy. Immunotherapy 2(5):609.

54. Zhu J, et al. (2015) Local administration of a novel Toll-like receptor 7 agonist in combination with doxorubicin induces durable tumouricidal effects in a murine model of T cell lymphoma. J Hematol Oncol 8:21.

55. Huang $\mathrm{H}$, et al. (2018) Reverse Screening Methods to Search for the Protein Targets of Chemopreventive Compounds. Front Chem 6:138.

56. Inaba K, et al. (1992) Generation of large numbers of dendritic cells from mouse bone marrow cultures supplemented with granulocyte/macrophage colony-stimulating factor. The Journal of experimental medicine 176(6):1693-1702.

57. Lutz MB, et al. (1999) An advanced culture method for generating large quantities of highly pure dendritic cells from mouse bone marrow. Journal of immunological methods 223(1):77-92.

58. Ho HH, et al. (2018) Vestibular schwannomas: Accuracy of tumor volume estimated by ice cream cone formula using thin-sliced MR images. PLOS ONE 13(2):e0192411.

59. Han Q, Wang Y, Pang M, \& Zhang J (2017) STAT3-blocked whole-cell hepatoma vaccine induces cellular and humoral immune response against HCC. J Exp Clin Cancer Res 36(1):156.

60. Cai H, Shukla S, Wang C, Masarapu H, \& Steinmetz NF (2019) Heterologous Prime-Boost Enhances the Antitumor Immune Response Elicited by Plant-Virus-Based Cancer Vaccine. J Am Chem Soc 141(16):6509-6518.

61. Ramesh A, Brouillard A, Kumar S, Nandi D, \& Kulkarni A (2020) Dual inhibition of CSF1R and MAPK pathways using supramolecular nanoparticles enhances macrophage immunotherapy. Biomaterials 227:119559.

62. Komohara Y, Fujiwara Y, Ohnishi K, \& Takeya M (2016) Tumor-associated macrophages: Potential therapeutic targets for anti-cancer therapy. Adv Drug Deliv Rev 99(Pt B):180-185. 
63. Huang XM, et al. (2014) Role of plasmacytoid dendritic cells and inducible costimulator-positive regulatory T cells in the immunosuppression microenvironment of gastric cancer. Cancer Sci 105(2):150-158.

64. Yu H, et al. (2013) Regulatory T cells and plasmacytoid dendritic cells contribute to the immune escape of papillary thyroid cancer coexisting with multinodular non-toxic goiter. Endocrine 44(1):172181.

65. Durham NM, et al. (2017) GITR ligand fusion protein agonist enhances the tumor antigen-specific CD8 T-cell response and leads to long-lasting memory. $J$ Immunother Cancer 5.

66. Crespo J, Sun H, Welling TH, Tian Z, \& Zou W (2013) T cell anergy, exhaustion, senescence, and stemness in the tumor microenvironment. Curr Opin Immuno/ 25(2):214-221.

67. Krishnamurti U, Wetherilt CS, Yang J, Peng LM, \& Li XX (2017) Tumor-infiltrating lymphocytes are significantly associated with better overall survival and disease-free survival in triple-negative but not estrogen receptor positive breast cancers. Hum Pathol 64:7-12.

68. Kimura T, et al. (2013) MUC1 Vaccine for Individuals with Advanced Adenoma of the Colon: A Cancer Immunoprevention Feasibility Study. Cancer Prev Res 6(1):18-26.

69. Bray F, et al. (2018) Global cancer statistics 2018: GLOBOCAN estimates of incidence and mortality worldwide for 36 cancers in 185 countries. Ca-Cancer J Clin 68(6):394-424.

70. Muinao T, Prasanna H, Boruah D, \& Pal M (2018) Diagnostic and Prognostic Biomarkers in ovarian cancer and the potential roles of cancer stem cells - An updated review. Exp Cell Res 362(1):1-10.

71. Zang RC, et al. (2019) Enhancement of diagnostic performance in lung cancers by combining CEA and CA125 with autoantibodies detection. Oncoimmunology 8(10).

72. Abbaszadegan MR, et al. (2017) Isolation, identification, and characterization of cancer stem cells: A review. J Cell Physiol 232(8):2008-2018.

73. Gordeeva $O$ (2018) Cancer-testis antigens: Unique cancer stem cell biomarkers and targets for cancer therapy. Semin Cancer Bio/ 53:75-89.

74. Zheng F, et al. (2018) Cancer Stem Cell Vaccination With PD-L1 and CTLA-4 Blockades Enhances the Eradication of Melanoma Stem Cells in a Mouse Tumor Model. J Immunother 41(8):361-368.

75. Danishefsky SJ, Shue YK, Chang MN, \& Wong CH (2015) Development of Globo-H cancer vaccine. Acc Chem Res 48(3):643-652.

76. Bowyer G, et al. (2018) Activation-induced Markers Detect Vaccine-Specific CD4(+) T Cell Responses Not Measured by Assays Conventionally Used in Clinical Trials. Vaccines (Basel) 6(3).

77. Cheung SK, et al. (2016) Stage-specific embryonic antigen-3 (SSEA-3) and beta3GalT5 are cancer specific and significant markers for breast cancer stem cells. Proc Natl Acad Sci U S A 113(4):960965.

78. Zhao F, et al. (2015) Cancer stem cell vaccine expressing ESAT-6-gpi and IL-21 inhibits melanoma growth and metastases. Am J Transl Res 7(10):1870-1882. 
79. Umar A (2014) Cancer Immunoprevention: A New Approach to Intercept Cancer Early. Cancer Prev Res 7(11):1067-1071.

\section{Tables}

Table 1. Summary of Patient Study Cohort Analyzed.

\begin{tabular}{|c|c|c|c|c|c|}
\hline Number & Sex & Age & Organization type & $\begin{array}{l}\text { OCT4 positive rate } \\
(\%)\end{array}$ & $\begin{array}{l}\text { Globo } \mathrm{H} \text { positive rate } \\
(\%)\end{array}$ \\
\hline 1 & male & 54 & Colorectal cancer & 10.35 & 25.69 \\
\hline 2 & female & 74 & Colorectal cancer & 25.31 & 34.16 \\
\hline 3 & male & & Sigmoid carcinoma & 38.94 & 54.39 \\
\hline 4 & female & 88 & Sigmoid carcinoma & 5.31 & 48.61 \\
\hline 5 & female & 73 & Litres of colon cancer & 7.59 & 23.17 \\
\hline 6 & male & 70 & Sigmoid carcinoma & 15.67 & 16.94 \\
\hline 7 & female & 48 & Colon cancer & 41.35 & 33.97 \\
\hline 8 & female & 85 & Colon cancer & 26.38 & 39.15 \\
\hline 9 & female & 60 & $\begin{array}{l}\text { Transverse colon } \\
\text { cancer }\end{array}$ & 9.84 & 42.92 \\
\hline 10 & female & 77 & Sigmoid carcinoma & 14.69 & 17.47 \\
\hline 11 & female & 67 & Sigmoid carcinoma & 29.46 & 24.65 \\
\hline 12 & female & 85 & Sigmoid carcinoma & 22.38 & 17.64 \\
\hline 13 & male & 73 & Right colon cancer & 34.17 & 28.97 \\
\hline 14 & male & 67 & Colorectal cancer & 17.32 & 34.91 \\
\hline 15 & male & 47 & Sigmoid carcinoma & 19.49 & 32.47 \\
\hline 16 & male & 68 & Right colon cancer & 5.88 & 28.41 \\
\hline
\end{tabular}

Analysis of samples from patients with colorectal cancer

\section{Figures}


A
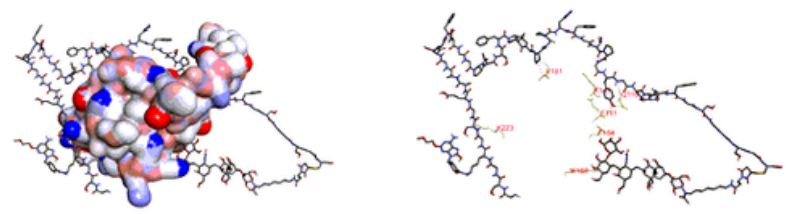

I
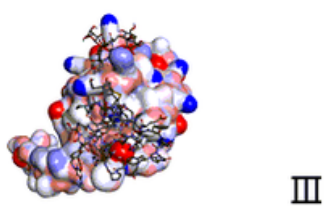

B

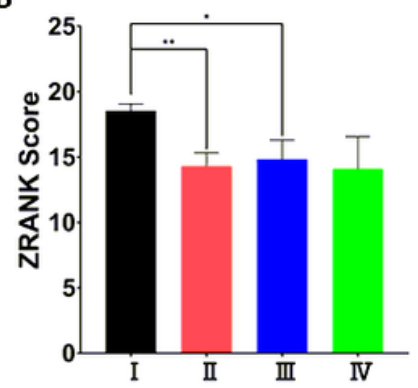

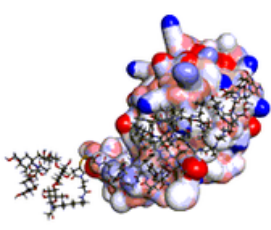

II

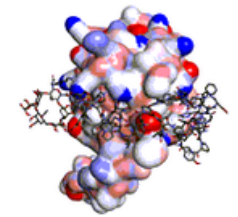

IV

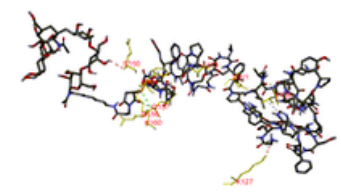

C

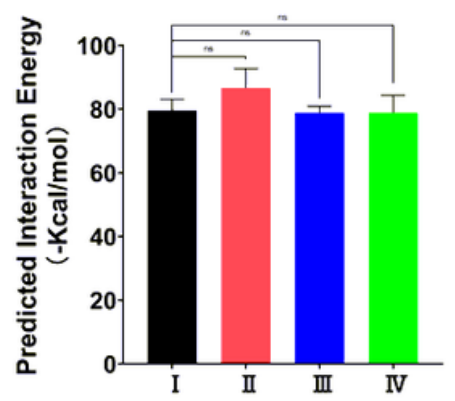

D
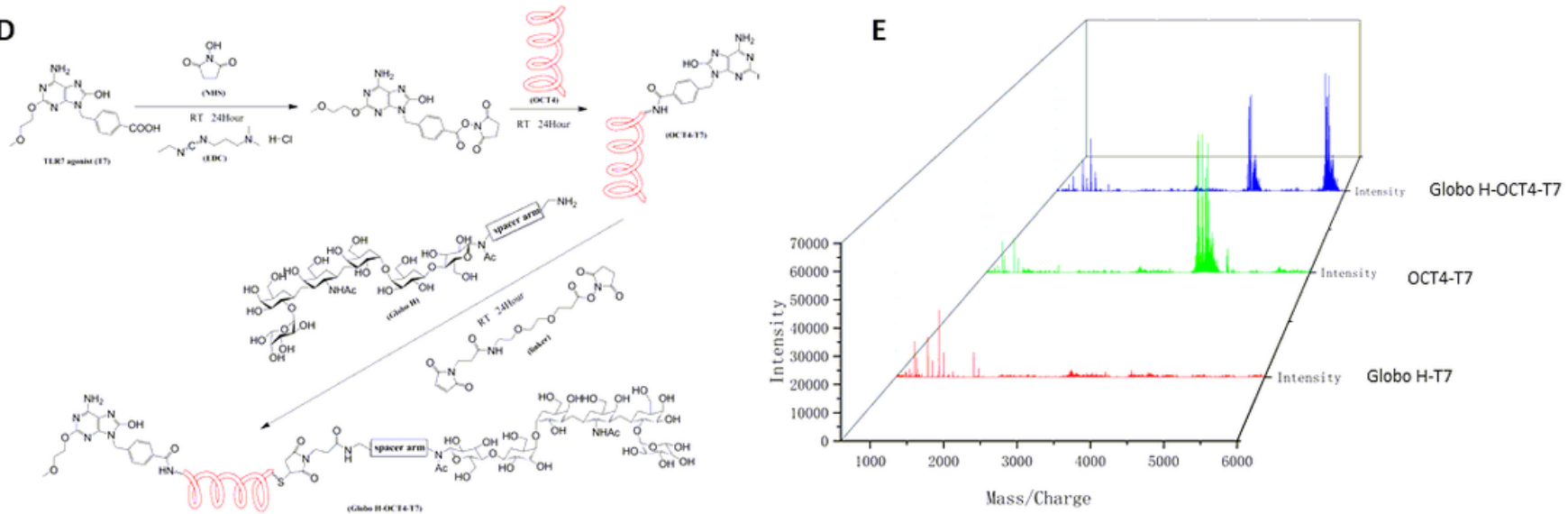

\section{Figure 1}

Preparation and characterization of Globo H-OCT4-T7. A: Homology modeling of DC-SIGN protein using Discovery studio software identifies a molecular docking pattern for four different vaccine structures combined with DC-SIGN. Green indicates hydrogen bonding and purple indicates conjugate weak interaction. B: Analysis of ZRANK SCORE in which four different vaccines were docked with DC-SIGN. C: Analysis of the energy binding of four different vaccines to DC-SIGN. D: The chemical synthesis route map of the vaccine. E: Mass spectrometry analysis of three vaccines, OCT4-T7, Globo H-T7 and Globo HOCT4-T7. *, $\mathrm{P}<0.05 ;$ **, $\mathrm{P}<0.01$. 


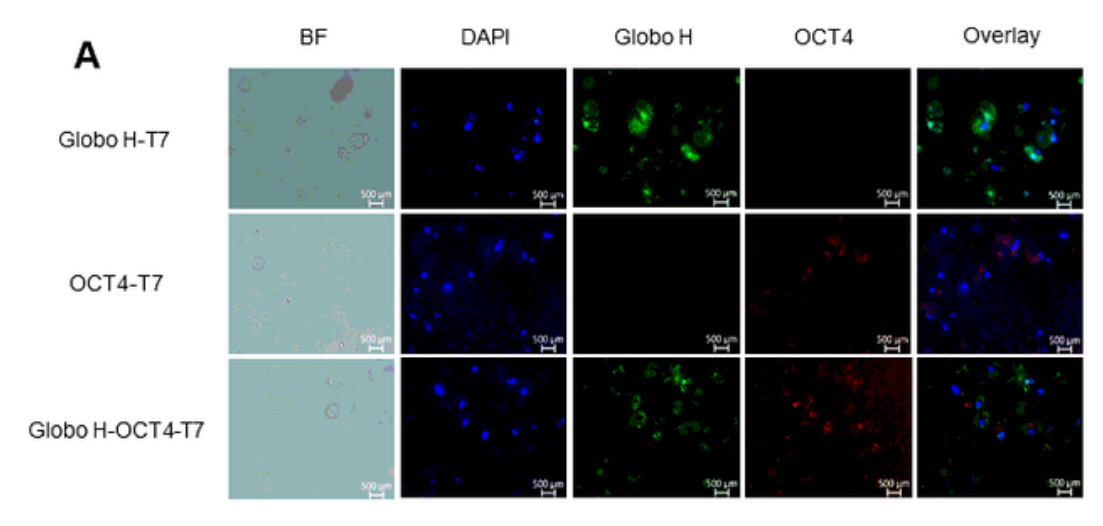

B

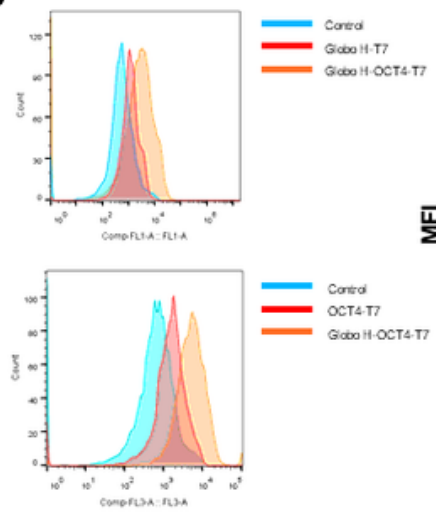

C

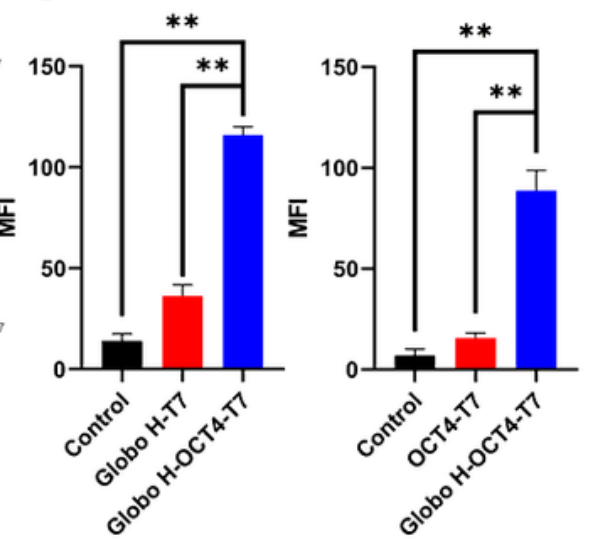

D
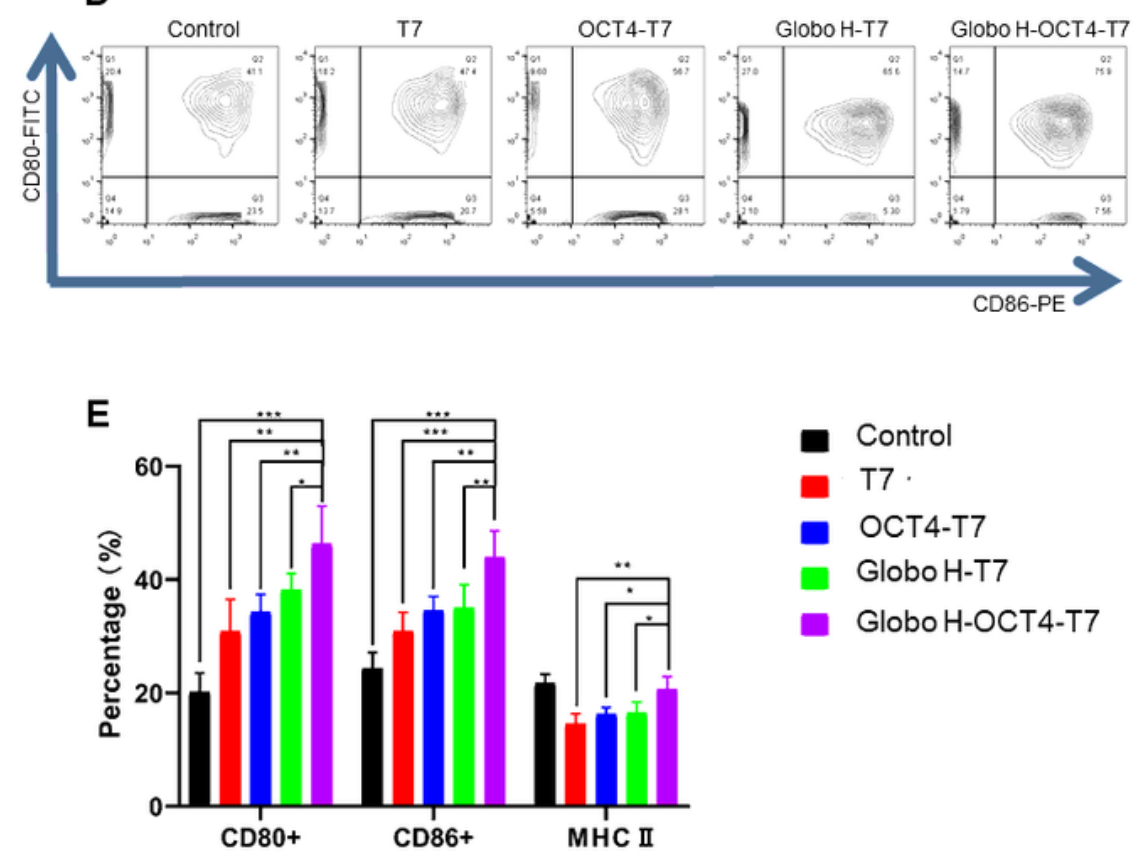

Figure 2

Globo H-OCT4-T7 promotes antigen uptake in BMDCs and induces BMDC maturation. A: Immunofluorescence detection of DC uptake of OCT4-T7, Globo H-T7 and Globo H-OCT4-TLR7.Green indicates Globo H; red indicates OCT4. B, C: FlowJo-based analyses of the fluorescence intensity of DC cells after uptake of the three vaccine antigens. D, E: BMDCs were stimulated with the OCT4-T7, Globo H- 
T7 and Globo H-OCT4-T7 vaccines, and DC surface maturation markers CD80, CD86, MHCll were detected by flow cytometry. ${ }^{*}, \mathrm{P}<0.05 ; * \star, \mathrm{P}<0.01 ; * \star *, \mathrm{P}<0.001$.
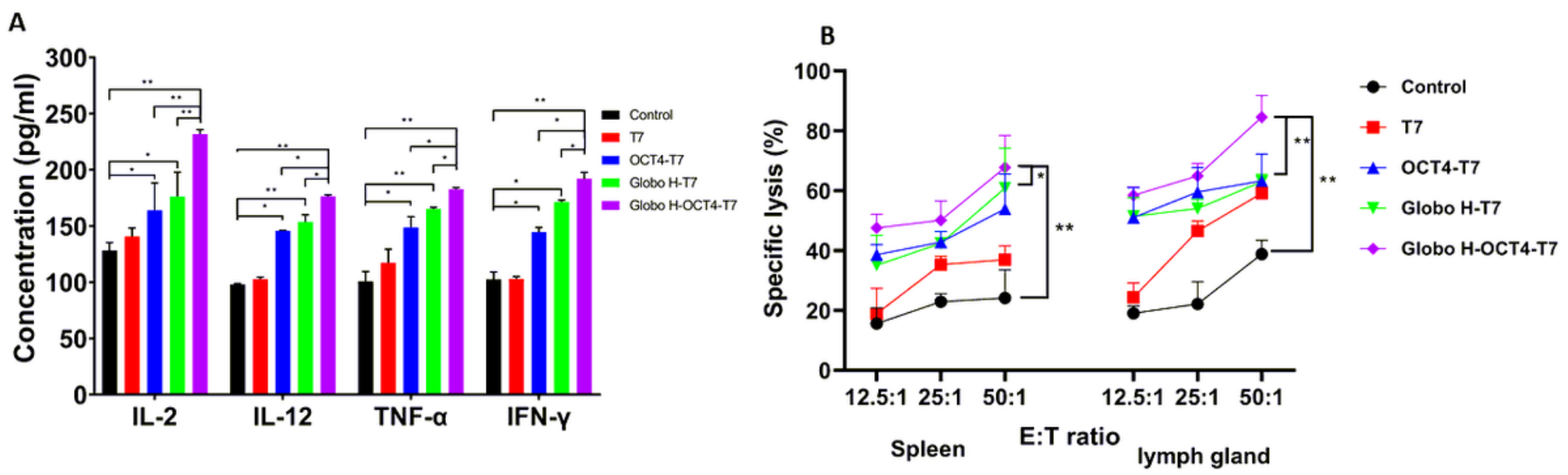

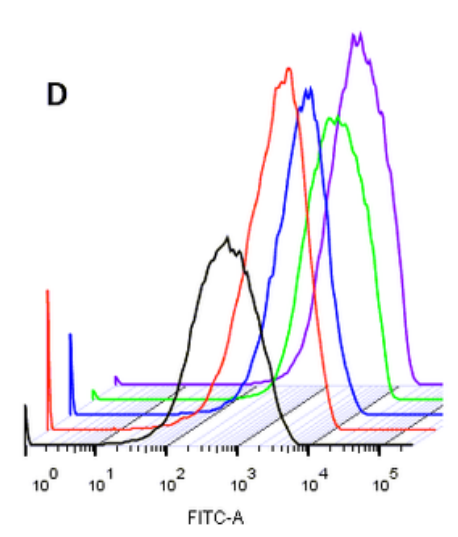

СT26

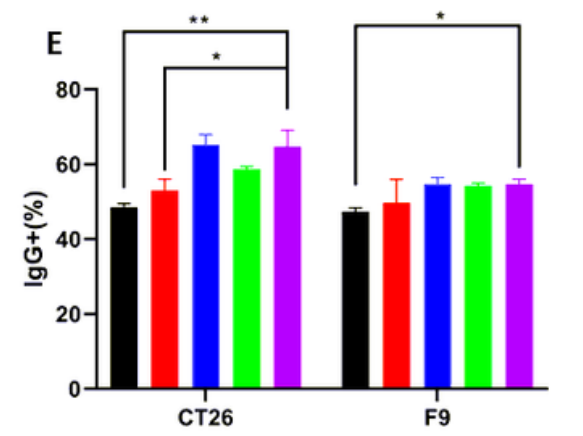

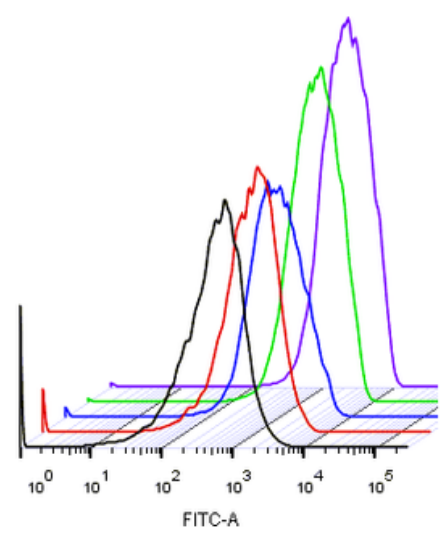

F9

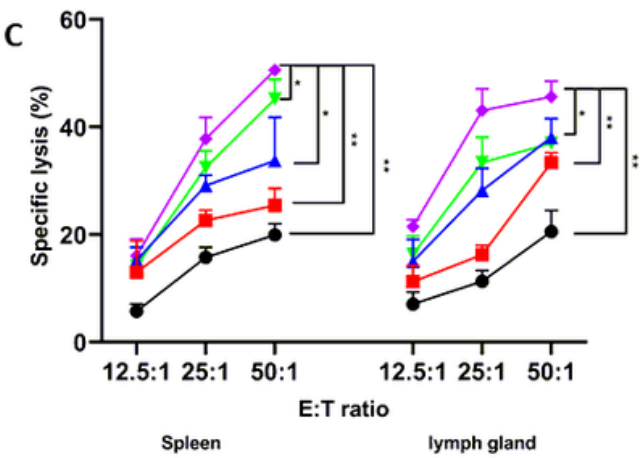

$\mathbf{F}$

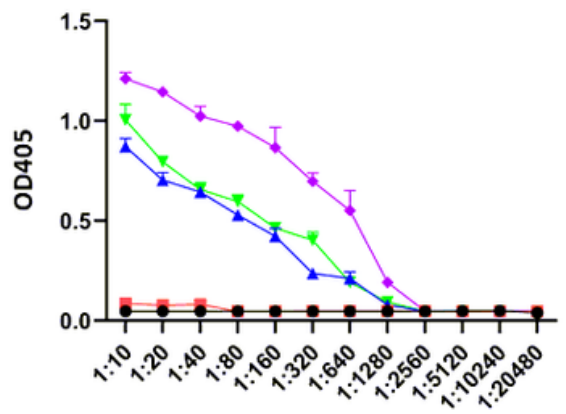

- Control

$-\mathrm{T} 7$

$\star$ OCT4-T7

$\rightarrow$ Globo H-T7

- Globo H-OCT4-T7

\section{Figure 3}

Globo H-OCT4-T7 induces the production of cytokines and enhances cytotoxic T lymphocytes (CTL) responses against the tumor. A: After four immunizations, serum was extracted from mice to determine the effects of different vaccines on IL-2, IL12, TNF-a, and IFN-y levels. B: The killing effect of cytotoxic lymphocytes after stimulation of different vaccines was examined. C: ELISA of the specific antibody content of OCT4 and Globo $\mathrm{H}$ after different vaccine stimulation. D, E: Flow-through detection of tumorspecific antibodies present in the serum after stimulation with different vaccines. ${ }^{*}, \mathrm{P}<0.05 ; * \star, P<0.01$. 


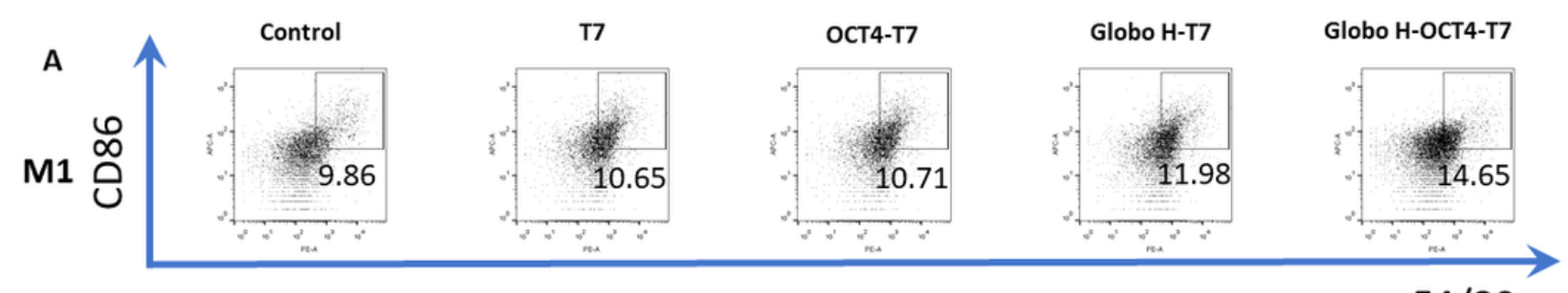

$\mathrm{F} 4 / 80$
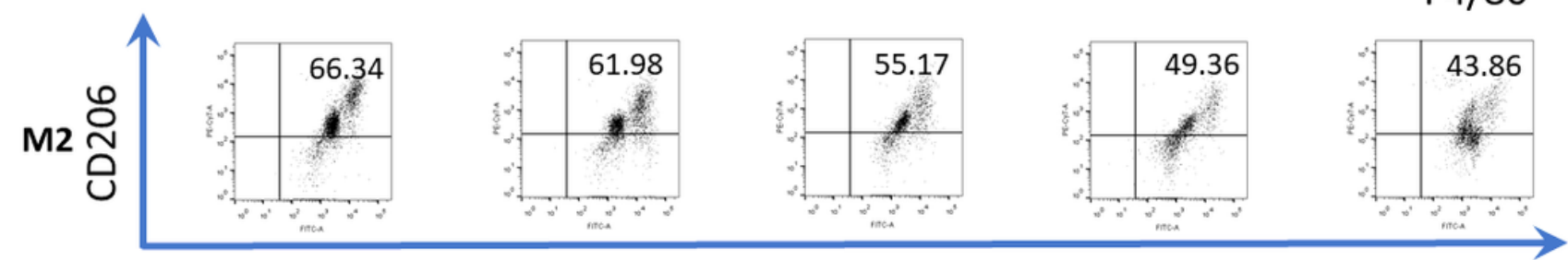

$\mathrm{F} 4 / 80 \mathrm{CD} 45$

B
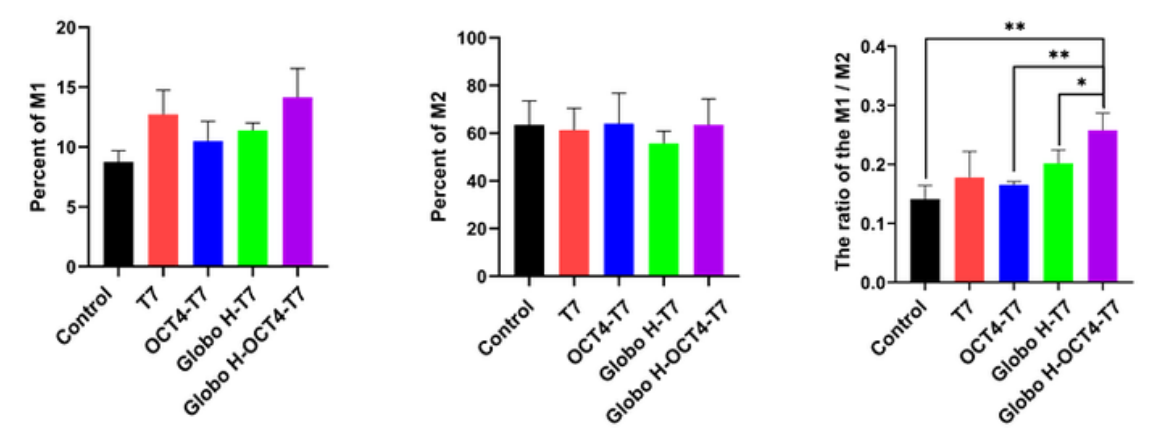

D

T7
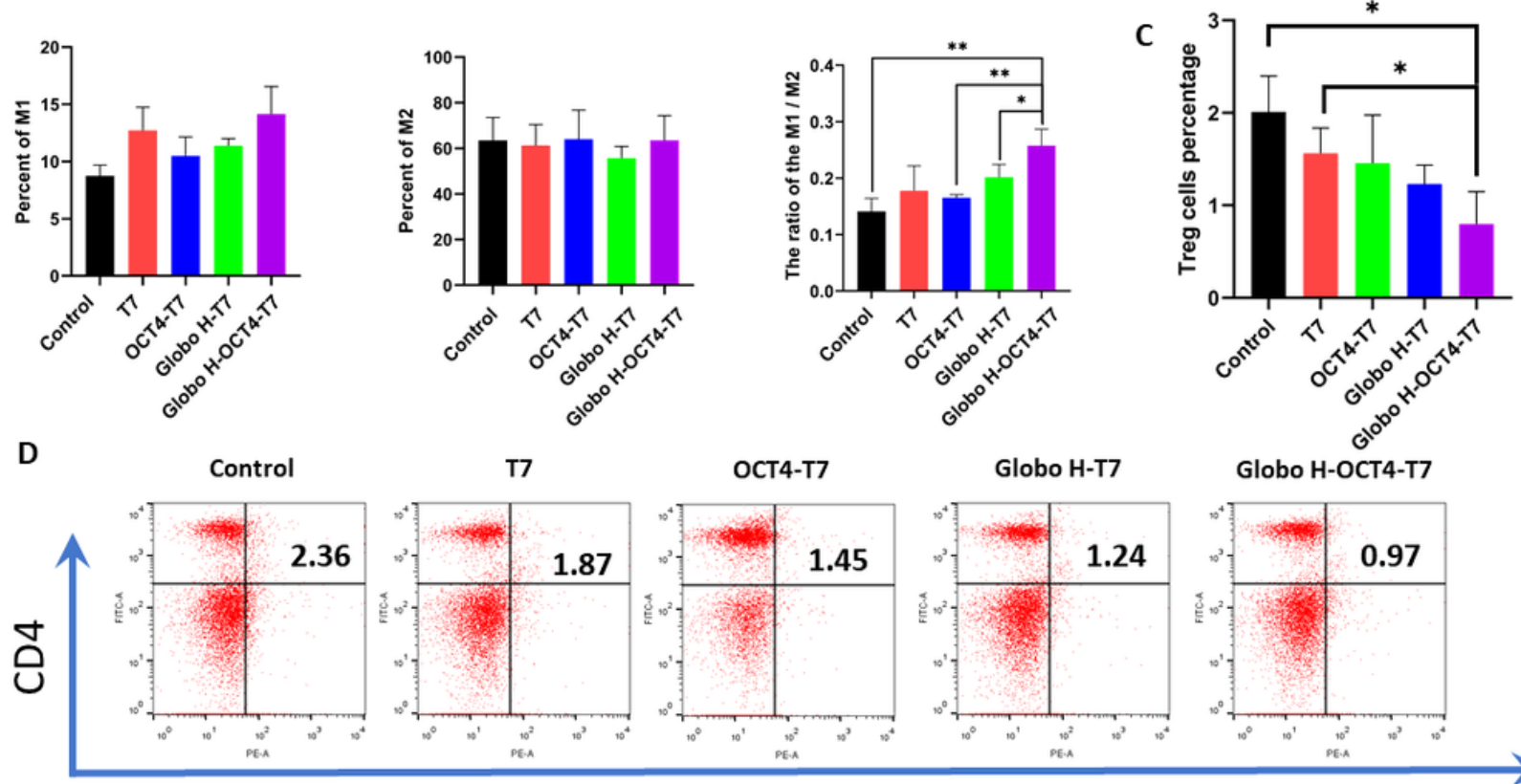

CD25 Foxp3

\section{Figure 4}

Globo H-OCT4-T7 has a therapeutic effect in a CT26 tumor model. A: Schematic representation of the dosing schedule for different vaccines in BALB/c mice. B, C: Tumor size on day 60 and tumor volume (mm3) was assessed every other day for 30 days after tumor implantation. D: Survival time in days of tumor-bearing mice after immunization with PBS, T7, OCT4-T7, Globo H-T7 or Globo H-OCT4-T7. **, P < $0.01 ; * \star \star, P<0.001$. 


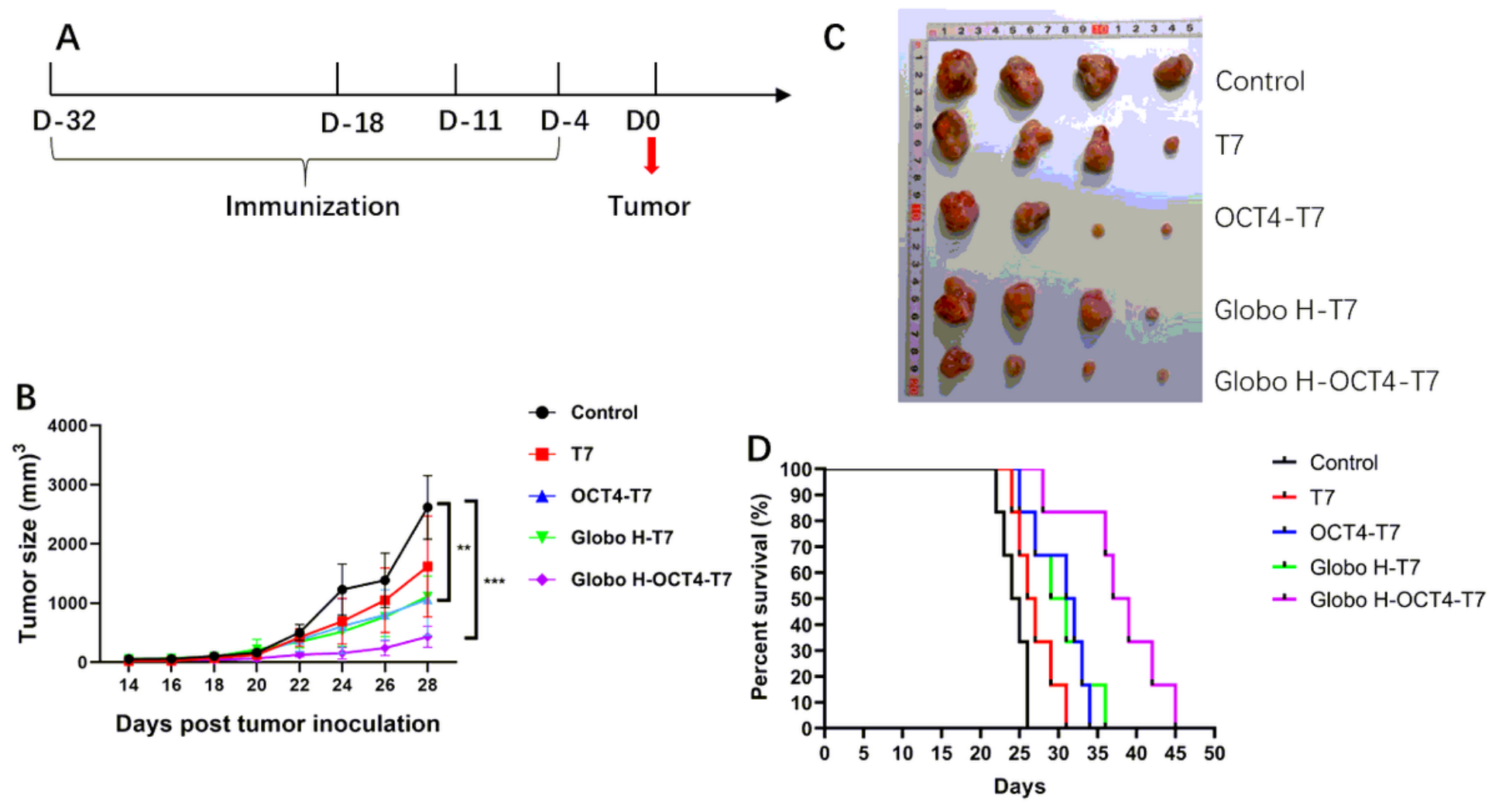

Figure 5

The Globo H-OCT4-T7 vaccine changes the proportion of macrophages in the tumor microenvironment and reduces regulatory T cells in PBMC cells. A, B: After four immunizations, $1 \times 105$ CT26 cells were subcutaneously implanted in mice, and the tumor-associated macrophages were isolated when the tumor was visible to the naked eye. The M1 macrophage surface markers CD86 and F4/80 and the M2 macrophage surface markers CD206, F4/80 and CD45 were analyzed by flow cytometry and their ratios were analyzed. C, D: PBMCs were isolated from mice after four immunizations, and the regulatory T-cell surface markers CD3, CD4, CD25 and Foxp3 were detected by flow cytometry. ${ }^{*}, \mathrm{P}<0.05 ;$;, $\mathrm{P}<0.01$. 
A

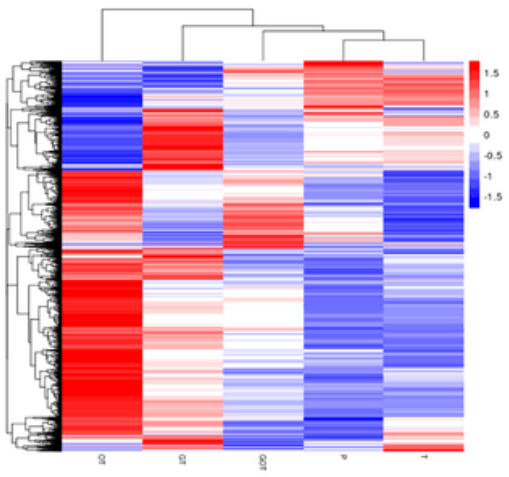

D

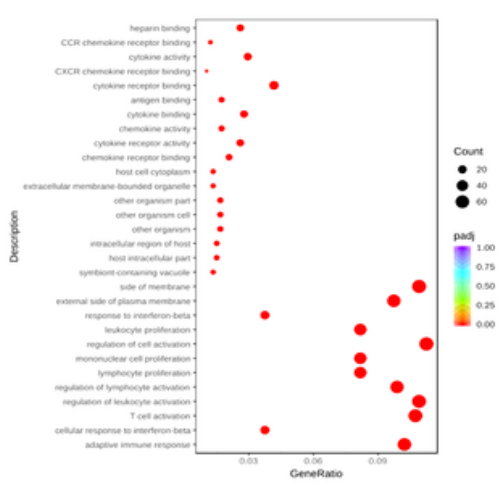

B

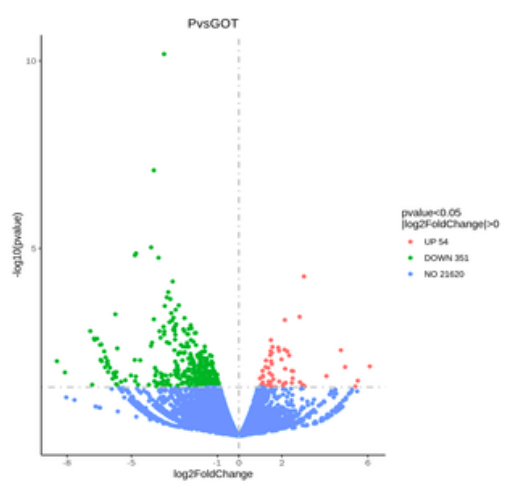

E

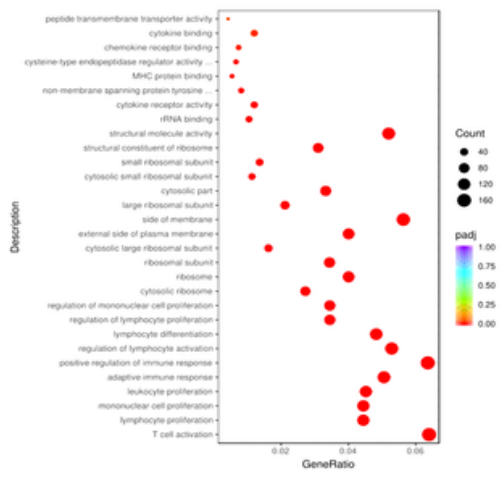

C

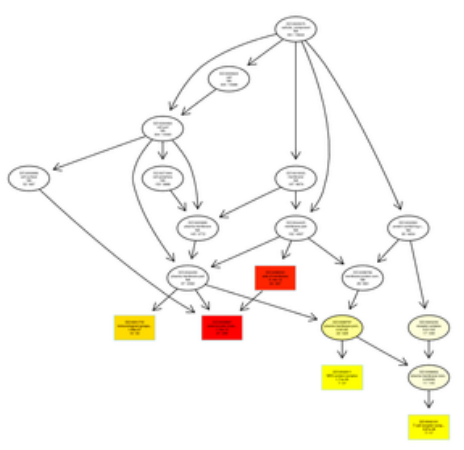

F

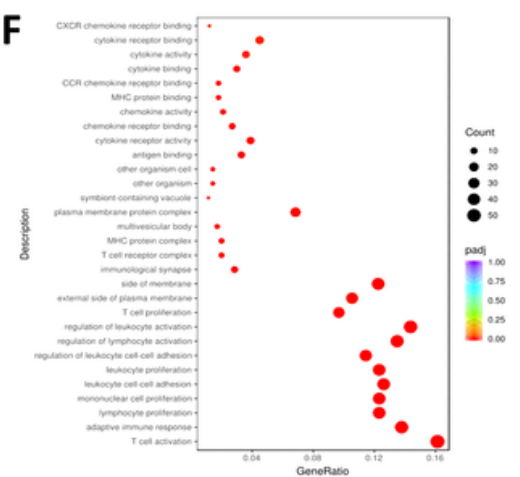

Figure 6

Gene expression analysis of tumor. A: Tumor gene heat maps of different groups of PBS, T7, OCT4-T7, Globo H-T7, and Globo H-OCT4-T7. B: transcriptome volcano map of the PBS and Globo H-OCT4-T7 groups; 54 genes were up-regulated and 351 genes were down-regulated. C: Path changes caused by Globo H-OCT4-T7. D-F: OCT4-T7 (D), Globo H-T7 (E), and Globo H-OCT4-T7 (F) gene enrichment maps compared to the PBS group, respectively. 

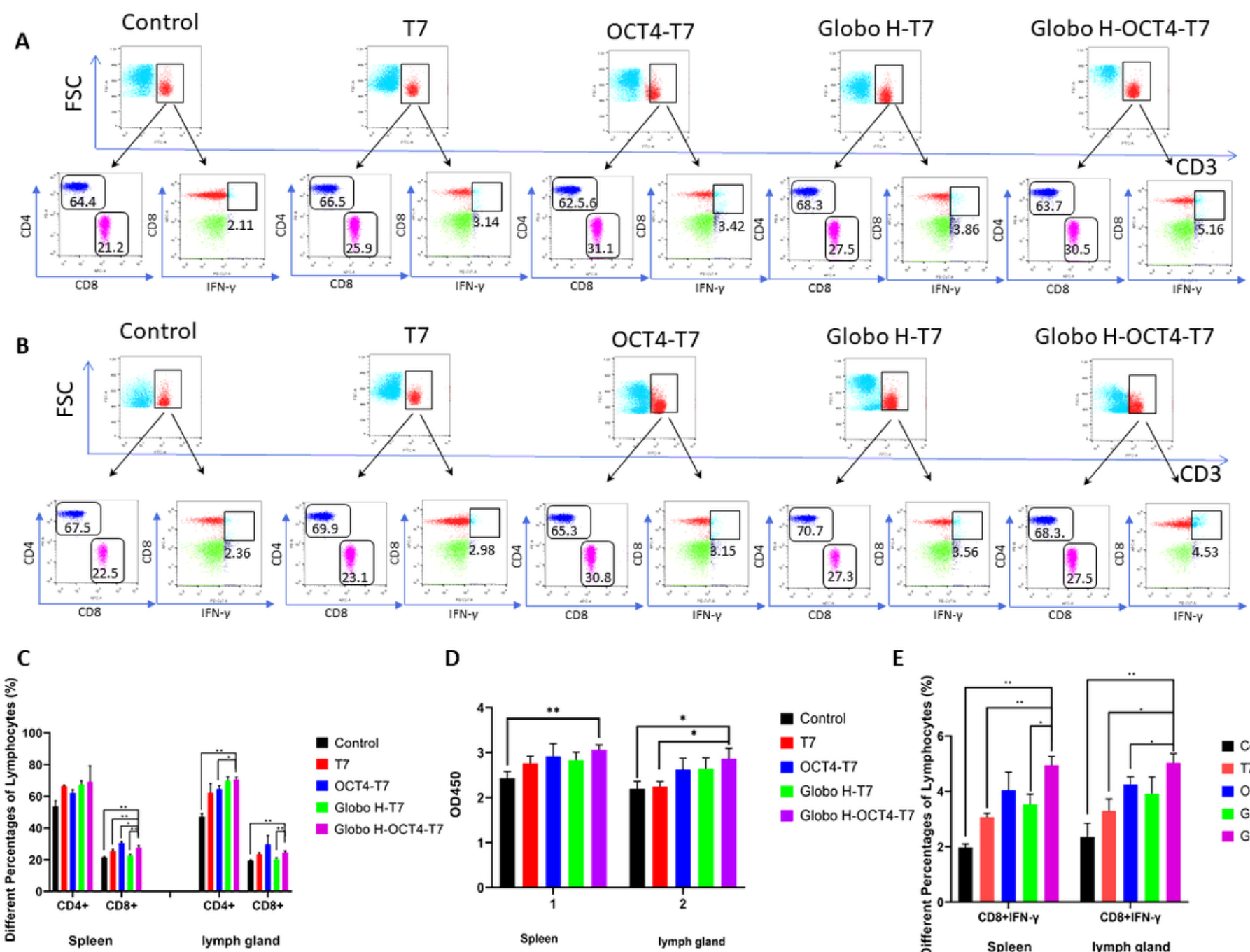

D

E
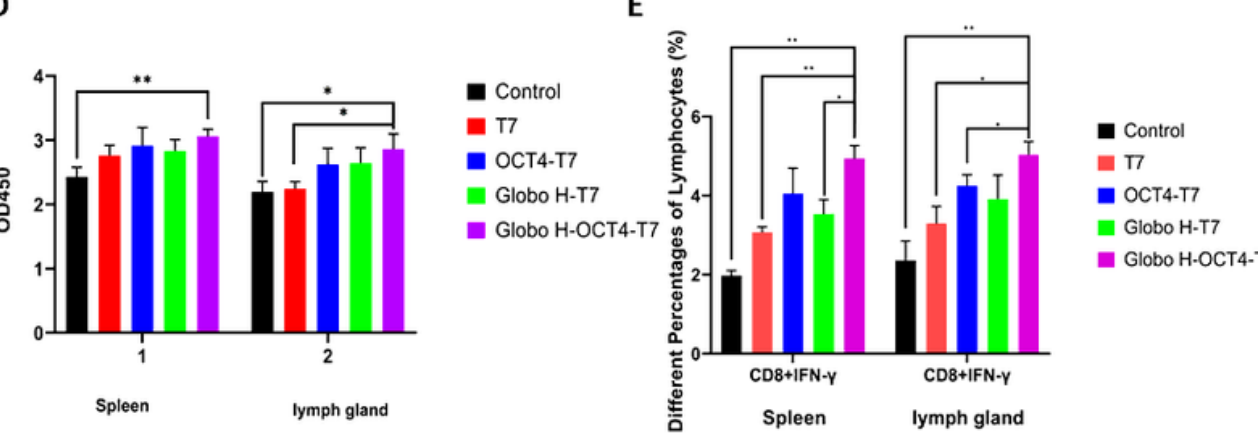

Figure 7

Globo H-OCT4-T7 as a combined vaccine exhibits a highly induced antitumor immune response. After four immunizations, the spleen and inguinal LNs were removed from mice and the lymphocytes were isolated (A, B). The content of CD3, CD4, CD8 and intracellular IFN- $y$ was detected by flow cytometry (C, E). D: MTT assay detects the proliferation of lymphocytes after in vitro stimulation by different vaccines. $\star, P<0.05 ; * \star, P<0.01$. 
A
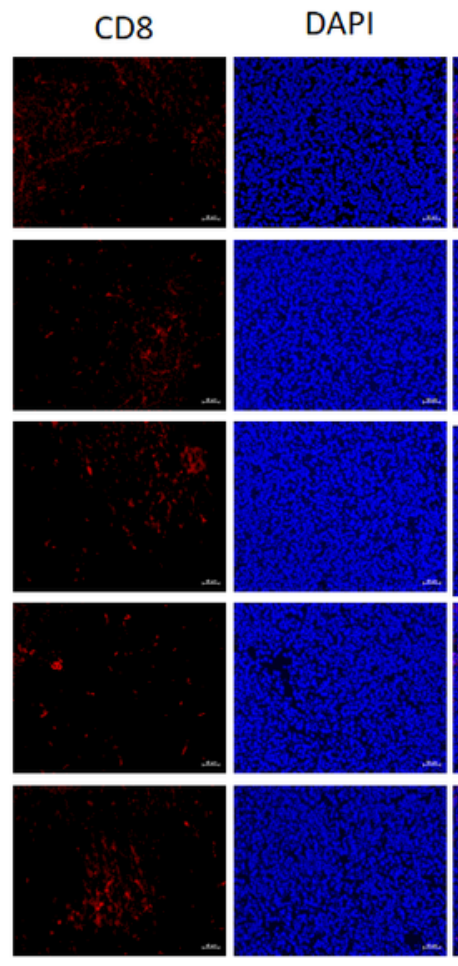

B

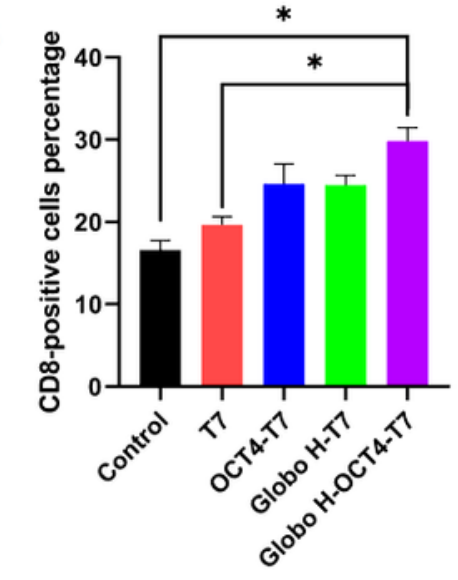

Merge
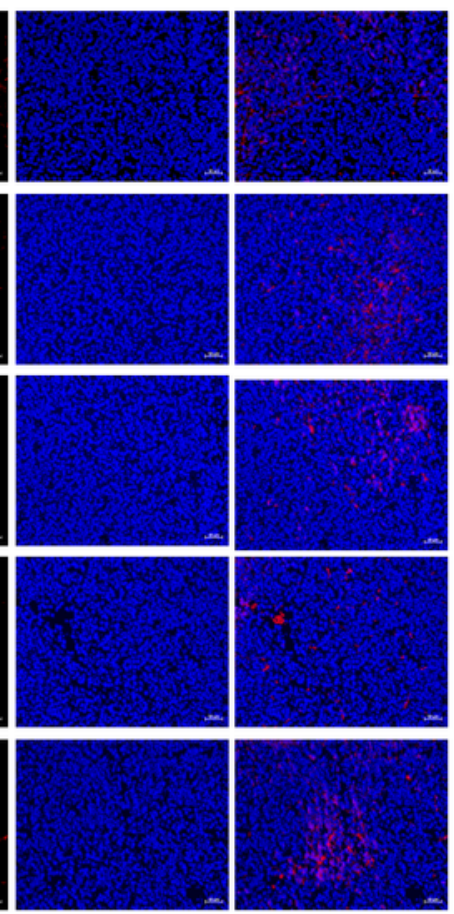

Globo H-OCT4-T7

Globo H-T7

OCT4-T7
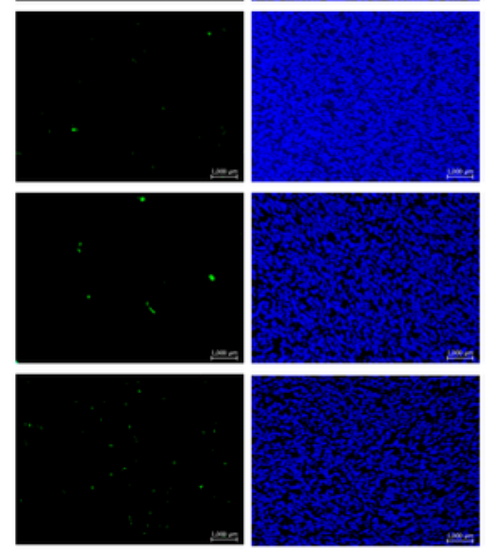

D

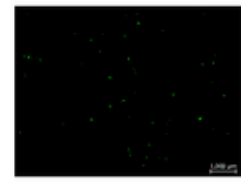

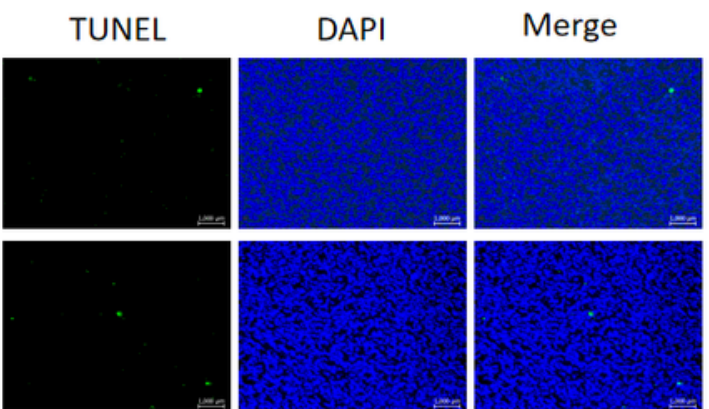
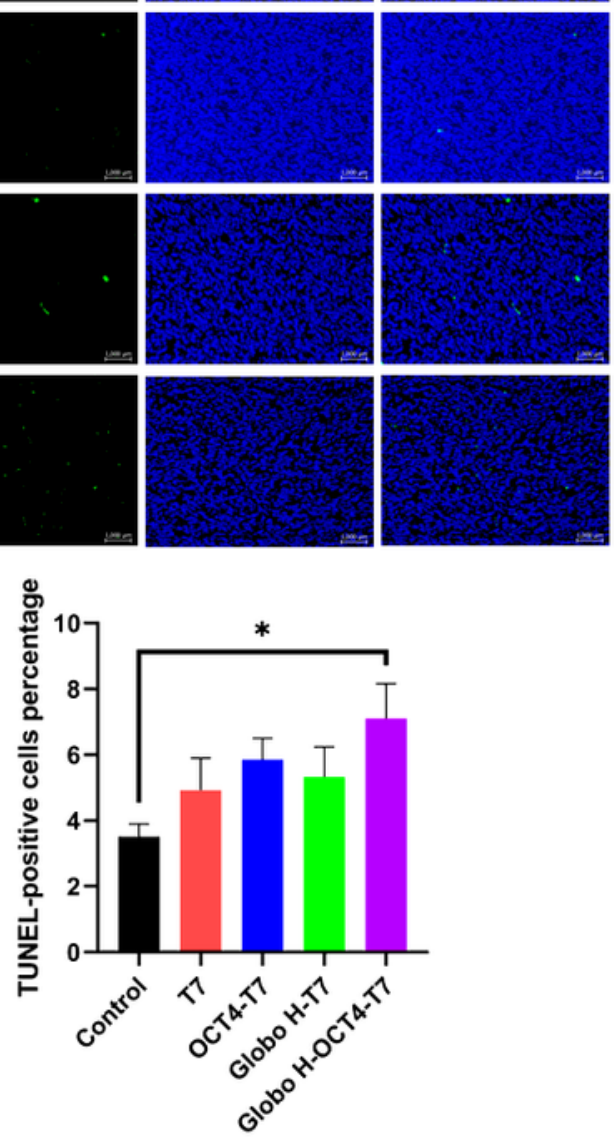

\section{Figure 8}

Globo H-OCT4-T7 increases the number of CD8+ cells in tumor microenvironment and promotes the apoptosis of tumor cells. After four immunizations, 1 x 105 CT26 cells were subcutaneously implanted into mice, and tumors were taken when the tumor was visible to the naked eye. Tumors were subjected to CD8 staining (A) and TUNEL (C), and the number of positive cells was recorded (B, D). * $P<0.05$. 
A

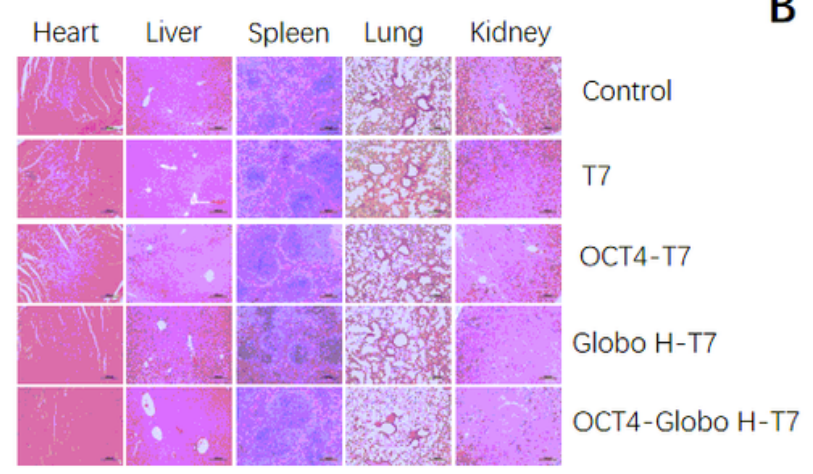

E

Before immunotherapy After immunotherapy

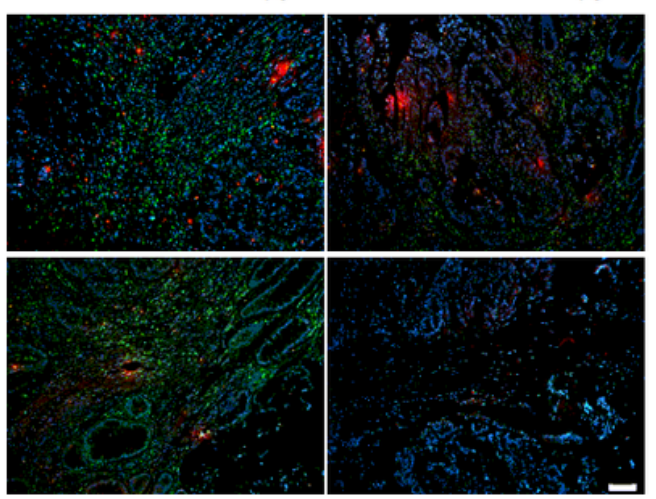

B

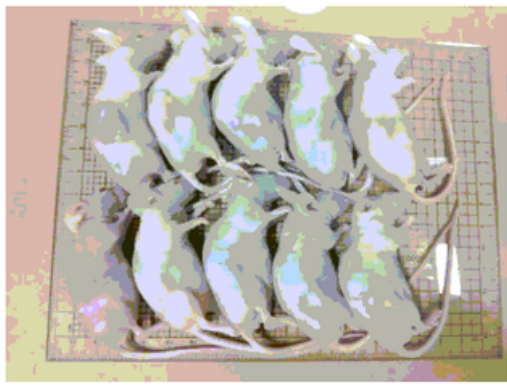

Control

$\mathbf{F}$

C

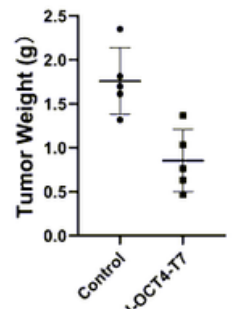

OCT4-Globo H-T7

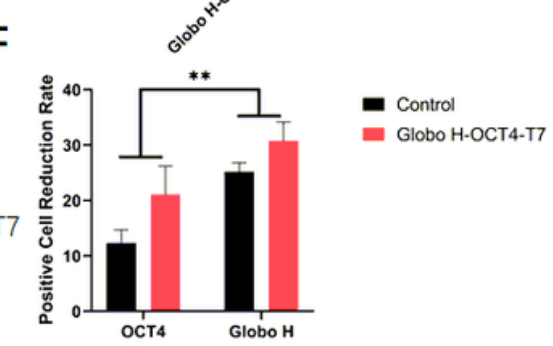

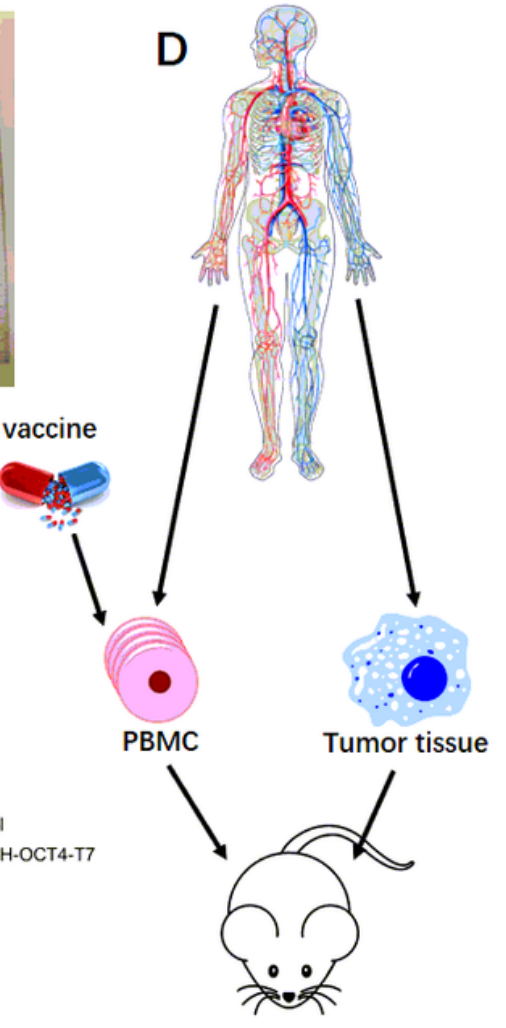

\section{Figure 9}

Globo H-OCT4-T7 is a safe method to inhibit tumor growth. A: H\&E staining was performed on the main organs of tumor-bearing mice, and pathological analysis was performed. D: Tumor tissues and PBMCs were isolated from patients with colorectal cancer, and 2x105 PBMCs were injected with different vaccines and injected into the PDX model. Tumor sizes are presented as (B) an image, and (C) tumor weight following euthanization and tumor isolation 50 days after treatment. E: Immunofluorescence staining of tumor tissue OCT4 and Globo H in the PDX model and homology patient tumor tissue. F: Calculate the percentage of positive cells. ${ }^{*}, \mathrm{P}$

\section{Supplementary Files}

This is a list of supplementary files associated with this preprint. Click to download.

- S1Table.docx 